\title{
Covariance Matrix Estimation with Non Uniform and Data Dependent Missing Observations
}

\author{
Eduardo Pavez Member, IEEE, and Antonio Ortega Fellow, IEEE
}

\begin{abstract}
In this paper we study covariance estimation with missing data. We consider missing data mechanisms that can be independent of the data, or have a time varying dependency. Additionally, observed variables may have arbitrary (non uniform) and dependent observation probabilities. For each mechanism, we construct an unbiased estimator and obtain bounds for the expected value of their estimation error in operator norm. Our bounds are equivalent, up to constant and logarithmic factors, to state of the art bounds for complete and uniform missing observations. Furthermore, for the more general non uniform and dependent cases, the proposed bounds are new or improve upon previous results. Our error estimates depend on quantities we call scaled effective rank, which generalize the effective rank to account for missing observations. All the estimators studied in this work have the same asymptotic convergence rate (up to logarithmic factors).
\end{abstract}

Index Terms-covariance estimation, missing data, effective rank.

\section{INTRODUCTION}

$\mathbf{T}$ HE covariance matrix is an essential component of many algorithms in machine learning [1], medical data analysis [2], [3], finance [4], and signal processing [5], [6], [7], [8], [9]. Estimation of covariance matrices involves the design and analysis of statistical procedures for recovering the covariance matrix from data samples. The prevalence and inevitability of missing data, along with the importance of covariance matrices in many applications and algorithms, makes the study of covariance estimation with missing observations of great importance.

When there are complete observations, the sample covariance matrix is the most commonly used estimator. Its performance is well understood under various settings (e.g., noise, high dimensions). A favored criteria to evaluate an estimator's performance is the estimation error measured in operator norm (largest singular value). This norm is preferred over other matrix norms (Frobenius, Schatten, etc.) because bounds for other norms imply operator norm bounds, while the converse is not true in general. The best estimation error bounds for the sample covariance matrix were recently obtained under sub-Gaussian [10], [11] and Gaussian [12] assumptions. Essentially, these results indicate that the number of samples required for accurate covariance estimation is proportional to the effective rank of the population covariance matrix, a parameter that is upper bounded by the rank, but that can be much smaller.

Authors are with the Department of Electrical and Computer Engineering, University of Southern California, Los Angeles, CA, 90089 USA. This work was funded in part by NSF under grants CCF-1410009 and CCF-2009032. (Author's e-mail: pavezcar@usc.edu, ortega@sipi.usc.edu)
Even though missing data is pervasive, and covariance matrices are utilized by a plethora of algorithms, studies on covariance estimation with missing data are few and of a narrower scope than those considering the complete data case. Perhaps because there are many available estimators, and each of them is designed and analyzed for a different type of missing data mechanism. In addition, mathematical expressions involving these estimators are much more intricate than those for complete observations. The works of [10], [13] stand out since they offer error analyses and bounds that match similar ones obtained for complete data. They studied convergence to the population covariance matrix (consistency) of different estimators by deriving finite sample error bounds. These works assume that the observations are independent and identically distributed (i.i.d.) copies of a sub-Gaussian vector, while the population covariance matrix may be structured or unstructured. Sparse and bandable structures were considered in [13], while [10] considered both structured (low rank) and unstructured covariance matrices. In addition, both papers assume the missing data mechanism is independent of the observations, however the specific assumptions and estimators are considerably different. More recently, [14] considered a non uniform and dependent missing data pattern. This work however, when simplified to the case studied by [10] (uniform independent observations), returns convergence rates that are sub-optimal with respect to the ambient dimension and the rate of missing entries.

In this work we do not make any structural assumptions (such as low rank or sparsity) on the population covariance matrix. We consider various missing data mechanisms and estimators, including a class of time varying dependent observations. Each of these mechanisms has a corresponding unbiased covariance estimator that generalizes the sample covariance matrix. For each case we provide bounds for the expected value of estimation error in operator norm. Our principal contributions are:

- We study existing covariance estimators under the missing completely at random (MCAR) missing data mechanism [15], which is data independent (see Section III). We consider an instance of this mechanism in which variables are observed according to dependent Bernoulli random variables with non uniform probabilities (the same missing data mechanism and estimator considered in [14]). We are the first to provide rate optimal error bounds (Theorem 1) for this setting. The case of equal observation probabilities (uniform case) was considered in [10], while the non uniform case with dependent observation 
variables was studied in [14].

- We consider another instance of the MCAR mechanism, in which the missing data distribution is unknown, therefore the observation probabilities have to be estimated from data. We propose an unbiased estimator that is closely related to the one used for estimation of structured covariance matrices [13], [16]. This work is the first in obtaining estimation error bounds in operator norm in the unstructured case (Theorem 2).

- We propose the conditionally MCAR (CMCAR) missing data mechanism, in which the missing data distribution evolves over time and depends on previous observations. We propose an unbiased estimator and characterize its estimation error (Theorem 3).

- Under certain conditions, we show that the bounds from Theorems 2 and 3, can be simplified to resemble the bound from Theorem 1 . This is possible because they all depend on quantities we call scaled effective rank, which generalize the effective rank to account for incomplete observations. In addition, after further simplifications, our bounds match or improve previous state of the art results with complete and missing observations.

This paper is organized as follows. In Section II we review the literature on covariance estimation with missing data. Section III introduces the missing data mechanisms and estimators. Error bounds are presented and discussed in Section IV, while numerical experiments are contained in Section V. Proofs and Conclusion appear in Sections VI and VII respectively.

\section{RELATED WORK}

\section{A. Applications of covariance matrices}

Missing data problems motivating this work are listed next. a) "Plug-in" estimators: Numerous algorithms use the sample covariance matrix as input. This "plug-in" principle can be used to extend these techniques to the missing data case by using estimators from this work that account for missing data. Examples that have followed this strategy include sparse sub-space clustering [17], multi-task learning [18], sub-space learning [19], classification [20], image compression [21], regression [22], principal component analysis [23], [24], and inverse covariance estimation [25], [16].

b) Applications with data acquisition costs: For some applications, obtaining complete observations might not be possible due to resource constraints. For example, in clinical studies, measuring certain features (e.g., acquiring samples or running tests) has monetary costs [26], [27], while in sensor networks obtaining measurements incurs in energy consumption [28], [29], [30]. For this reason, data may be acquired based on the cost of acquiring each sample, as well as information from previous observations. In these examples a covariance matrix may be required for statistical analysis, but standard covariance estimators are not well suited since observations are incomplete, possibly non i.i.d., and the missing data mechanism may evolve over time.

c) Statistical estimation and inference with partial observations: A very high ambient dimension can be problematic for data storage, transmission, or processing. But, since most natural signals are very structured, a few partial observations are often sufficient for performing simple statistical tasks like estimation and detection [31]. Covariance estimation from compressed measurements has been studied for structured [32], [33], and unstructured covariance matrices [34], [35], [36], [37], [38]. Additionally, active learning approaches, have been shown to benefit sequential hypothesis testing [39], Gaussian graphical model selection [40], [41], and covariance estimation [42].

\section{B. Covariance estimation with complete observations}

The complete data is denoted by the $n \times N$ matrix $\mathbf{X}$, where the columns of $\mathbf{X}$ are assumed to be zero mean, independent and identically distributed (i.i.d.) realizations of a vector $\mathbf{x}$ with population covariance matrix $\Sigma$. The starting point of most covariance estimation studies is the sample covariance matrix given by

$$
\mathbf{S}=\frac{1}{N} \mathbf{X X}^{\top}
$$

which is an unbiased estimator for the population covariance matrix $\boldsymbol{\Sigma}$, that is, $\mathbb{E}[\mathbf{S}]=\boldsymbol{\Sigma}$. Some recent papers have shown that the estimation error in operator norm of the sample covariance matrix is characterized by the effective rank of $\boldsymbol{\Sigma}$ [10], [11], [12]. These results imply the expectation bound

$$
\frac{\mathbb{E}[\|\boldsymbol{\Sigma}-\mathbf{S}\|]}{\|\boldsymbol{\Sigma}\|} \leq \mathcal{O}\left(\sqrt{\frac{\mathrm{r}(\boldsymbol{\Sigma})}{N}} \vee \frac{\mathrm{r}(\boldsymbol{\Sigma})}{N}\right),
$$

where $a \vee b$ stands for $\max (a, b)$. The $\mathcal{O}$ notation hides logarithmic dependencies on the dimension $n$ and number of samples $N$. The effective rank $\operatorname{r}(\boldsymbol{\Sigma})$ (see Definition 1) is upper bounded by the actual rank, but can be much smaller, thus offering a more nuanced measure of dimensionality.

Inequality (2) implies that the sample complexity of (1) is $N=\mathcal{O}\left(\operatorname{r}(\boldsymbol{\Sigma}) / \epsilon^{2}\right)$, that is, the number of i.i.d. samples required for the error in (2) to be below $\epsilon$ is proportional to the effective rank. Results from [11], [10] assume a sub-Gaussian distribution, while [12] provides dimension free bounds (without logarithmic dependence on $n$ ) for the Gaussian case. Our bounds are also based on the effective rank, so they can be compared with results that assume complete observations.

\section{Missing data mechanisms}

Construction of unbiased estimators requires knowledge of the missing data mechanism, i.e., the process by which data is lost. Recent literature of covariance estimation with missing observations considers mechanisms that are data independent. This type of mechanism is called missing completely at random (MCAR) [15]. We also introduce a new one called conditionally MCAR (CMCAR).

The MCAR mechanism has been used to model missing features in machine learning applications [43], or data loss in sensor networks, where transmission errors (e.g., packet loss), or sensor failures induce missing data. This mechanism has also been used to model incomplete observations in clinical and longitudinal studies [44], in which data is lost due to subject dropout. When the missing data is MCAR, we study 
two scenarios, known and unknown missing data distribution. In the first case, coordinates are observed according to dependent Bernoulli random variables that may have different probabilities. A value $0 / 1$ indicates a missing/observed variable. Covariance estimators designed for this setting appear in [10], [22], [14]. In [10], the missing data mechanism is modeled by independent Bernoulli variables with uniform distribution (all coordinates are observed with the same probability), while [14] addresses the dependent non uniform case. In [22], the independent case was considered, and the covariance matrix estimator was not analyzed directly, but as part of a linear regression study. In the second case, when the missing data distribution is MCAR but unknown, we compute estimates of the observation probabilities which can be used to construct an unbiased estimator. This estimator is the more natural generalization of the sample covariance matrix, and to the best of our knowledge, there are no available results for its estimation error. A similar approach, however rendering biased consistent estimators, has been used in [16], [13] as a component of other procedures.

We also consider a new missing data mechanism, which we call conditionally MCAR (CMCAR). For this case, coordinates are observed according to dependent Bernoulli random variables, but their distributions can change over time, and depend on previous observations. A simplified version of this mechanism was proposed in [42], however they used a different estimator, and no error bounds were provided. The CMCAR mechanism is closely related to active learning, which are data adaptive procedures designed to maximize system performance, while complying with problem specific cost constraints. This mechanism can model situations occurring in sensor networks, where data dependent missing observations may occur if the signal being measured can affect the sensing process. For example, temperature and humidity may damage a sensor over time. In other applications, if an observer is seeking to improve system performance, the subset of variables that are acquired/missed may depend on the data. In some scenarios, data acquisition carries a cost and obtaining complete observations might not be possible. For example, in clinical studies, measuring certain features (e.g. acquiring samples or running tests) may be expensive [26], [27], or in sensor networks, obtaining measurements incurs in energy consumption [28], [29], [30]. Active feature acquisition techniques have been applied to reduce the cost of predictive and classification algorithms while maintaining performance [45], [46], [47], [48].

\section{Covariance estimation with missing data}

The MCAR mechanism has been considered in previous statistical studies involving the covariance matrix [22], [10]. Particularly in [10], the author considers MCAR observations with uniform Bernoulli distribution, i.e., all variables are observed with probability $p>0$, and the estimator of (3). That study shows that the error bound and sample complexity depend on $\mathrm{r}(\boldsymbol{\Sigma}) / p^{2}$, thus effectively requiring $1 / p^{2}$ more samples than the sample covariance matrix to achieve the same performance. That work also proposes a regularized estimator for low rank covariance matrices, and shows that by carefully choosing a regularization parameter, its estimation error attains optimal error rates. A more recent work [14] obtained concentration bounds for the error measured in operator norm when the observations are characterized by dependent Bernoulli random variables with arbitrary observation probabilities. Altough that work is the first to consider the non uniform dependent setting, their error bounds have sub-optimal dependencies on the ambient dimension (an additional linear factor), and the fraction of missing entries (see Table I and Section III for more details).

Cai and Zhang [13] studied estimation of sparse and bandable covariance matrices under a MCAR missing data mechanism. The obtained error bounds match those for complete observations, up to scale factors that reflect the effect of missing data. Additionally, the error bounds are pessimistic, since they depend only on the least observed entry of the covariance matrix. Neither study [10], [13] offers insight into the relations among the missing data mechanism, the entries of the population covariance matrix and the covariance estimation error. This is because the missing data mechanism is too simple [10], or the error bounds are too conservative [13]. Other works have focused on estimation of sparse inverse covariance matrices [22], [16]. In particular [22] derives an error bound for the covariance matrix that is valid for sparse Gaussian graphical models. Missing data in principal component analysis was considered in [24]. Table I lists the most relevant covariance estimators and compares them in terms of sample complexity, that is, the minimum number of observations required to achieve error below $\epsilon$. A particular version of the CMCAR mechanism and a covariance estimator was introduced in [42], but no error bounds were provided.

\section{COVARIANCE ESTIMATORS}

\section{A. Notation}

We denote matrices and vectors by bold letters. For a matrix $\mathbf{X}=\left(x_{i j}\right)$ its entries are denoted by $x_{i j}$ or $\mathbf{X}_{i j}$ and for a vector $\mathrm{x}$ its $\mathrm{i}$-th entry is denoted by $x_{i}$. The entry-wise product $^{1}$ between matrices is defined as $(\mathbf{A} \odot \mathbf{B})_{i j}=a_{i j} b_{i j}$. We use $\|\cdot\|_{q}$ for entry-wise matrix norms, with $q=2$ corresponding to the Frobenius norm. $\|\cdot\|$ denotes the $\ell_{2}$ norm when applied to vectors, and the operator norm (largest singular value) when applied to matrices. The nuclear norm (sum of singular values) is denoted by $\|\cdot\|_{\star}$. The set of integers $\{1,2, \cdots, d\}$ is abbreviated by $[d]$.

\section{B. Missing observations}

The complete data is represented with the $n \times N$ matrix $\mathbf{X}$. Its $i$-th row and $k$-th column are the vectors $\mathbf{x}_{i}$ and $\mathbf{x}^{(k)}$ respectively. The $n \times N$ matrices $\mathbf{Y}$ and $\boldsymbol{\Delta}$ denote the observation and missing data patterns respectively. $\boldsymbol{\Delta}$ has entries in $\{0,1\}$. The $k$ th columns of $\mathbf{Y}$ and $\boldsymbol{\Delta}$ are denoted by the vectors $\mathbf{y}^{(k)}$ and $\boldsymbol{\delta}^{(k)}$ respectively. These quantities are related through

$$
\mathbf{y}^{(k)}=\boldsymbol{\delta}^{(k)} \odot \mathbf{x}^{(k)}
$$

\footnotetext{
${ }^{1}$ The entry-wise product is also known as Hadamard or Schur product.
} 


\begin{tabular}{|l|l|l|l|l|l|}
\hline Paper & Structure & Estimator & Missing data & Sample complexity (uniform case) \\
\hline Bunea \& Xiao [11] & any & sample covariance & N/A & $\mathrm{r}(\boldsymbol{\Sigma}) \log (n) / \epsilon^{2}$ \\
\hline Cai \& Zhang [13] & bandable & block threshold & MCAR & $\left(1 / \widehat{p}_{\min }\right)\left(C \log (n) / \epsilon^{2} \vee\left(C / \epsilon^{2}\right)^{1+1 / 2 \alpha}\right)$ \\
Cai \& Zhang [13] & sparse & adaptive threshold & MCAR & $\rho_{n, N} \log (n) / \epsilon^{2}$ \\
Lounici [10] & any & Eq.(3) & UI MCAR & $\left(\mathrm{r}(\boldsymbol{\Sigma}) / p^{2}\right) \log (2 n)\left(1 / \epsilon \vee\left(\log (n)+\log (N)+c_{1} p\right)\right)^{2}$ \\
Park \& Lim [14] & any & Eq.(3) & NUD MCAR & $\left(\mathrm{r}(\boldsymbol{\Sigma}) / p^{1 / 2}\right) \log (2 n)\left(n / p^{3 / 2} \epsilon \vee\left(\log (n)+\log (N)+c_{1} p\right)\right)^{2}$ \\
\hline Theorem 1 & any & Eq.(3) & NUD MCAR & $\left(\mathrm{r}(\boldsymbol{\Sigma}) / p^{2}\right) \log (n)\left(e \log (N) \vee 2 C_{2} / \epsilon\right)^{2}$ \\
\hline Theorem 2 & any & Eq.(5) & MCAR & $\mathrm{r}_{\min }(\boldsymbol{\Sigma}, \widehat{\mathbf{P}}) \log (n)\left(e \rho \log (N) \vee 2 C_{2} / \epsilon\right)^{2}$ \\
\hline
\end{tabular}

TABLE I: Comparison of covariance estimators with missing data. For Cai \& Zhang [13], the constants $\alpha$ and $\rho_{n, N}$ parameterize the classes of bandable and sparse covariance matrices respectively. $\widehat{p}_{\min }$ is the smallest non zero entry of $\widehat{\mathbf{P}}$. Some universal numerical constants have been ignored, for detailed results see corresponding Theorems in Section IV. Abbreviations: uniform independent (UI), non uniform dependent (NUD), expectation bound (E), high probability bound (P).

When the $j$-th entry of $\boldsymbol{\delta}^{(k)}$ is equal to 0 , the corresponding entry of $\mathbf{x}^{(k)}$ is missing. If the column vectors $\mathbf{x}^{(k)}$ are i.i.d., zero mean and with population covariance matrix $\Sigma$, the sample covariance matrix of the complete data

$$
\mathbf{S}=\frac{1}{N} \mathbf{X X}^{\top}=\frac{1}{N} \sum_{k=1}^{N} \mathbf{x}^{(k)} \mathbf{x}^{(k)^{\top}},
$$

is an unbiased estimator for $\boldsymbol{\Sigma}$. The behavior of $\mathbf{S}$ for finite $N$ has been studied extensively and it is well understood [10], [11], [12]. In the rest of this section we will introduce alternative estimators for $\boldsymbol{\Sigma}$ that depend on the observations $\mathbf{Y}$, and properties of the missing data pattern $\boldsymbol{\Delta}$.

\section{Missing data mechanisms and covariance estimators}

Consider a random vector $\mathbf{x}=\left[x_{1}, x_{2}, \cdots, x_{n}\right]^{\top}$ in $\mathbb{R}^{n}$ with population covariance matrix $\boldsymbol{\Sigma}$. The purpose of a covariance estimator is to accurately approximate $\boldsymbol{\Sigma}$ from $\left\{\mathbf{x}^{(k)}\right\}_{k=1}^{N}$, a set of independent identically distributed (i.i.d.) copies of $\mathbf{x}$. Here, however, we only have access to the sequence of incomplete observations $\left\{\mathbf{y}^{(k)}\right\}_{k=1}^{N}$. Our interest is in estimators with the following desirable properties:

1) Unbiased: their expected value is equal to $\boldsymbol{\Sigma}$.

2) Consistent: as the number of observations $N \rightarrow \infty$ the estimator converges to $\boldsymbol{\Sigma}$.

3) Minimum sample complexity: minimum number of samples to achieve error below $\epsilon>0$.

We consider two missing data mechanisms, and three different estimators which are described next.

a) Unbiased estimator under MCAR observations with known observation probabilities: The MCAR model asserts that $\mathbf{X}$ and $\boldsymbol{\Delta}$ are independent [15]. We model the missing data with a random vector $\boldsymbol{\delta}=\left(\delta_{i}\right)$ of dependent Bernoulli $0-1$ random variables, where $\mathbb{P}\left(\delta_{i}=1, \delta_{j}=1\right)=\mathbb{E}\left[\delta_{i} \delta_{j}\right]=p_{i j}$. We assume that $\boldsymbol{\delta}^{(k)}$ are i.i.d. copies of $\boldsymbol{\delta}$. If all $p_{i i}=1$, then $\mathbf{y}^{(k)}=\mathbf{x}^{(k)}$ for all $k$, thus we have perfect observation of $\mathbf{x}^{(k)}$. We are interested in recovering the covariance matrix of $\mathbf{x}$ from $\left\{\mathbf{y}^{(k)}\right\}_{k=1}^{N}$, when $0<p_{i j} \leq 1$ for all $i \in[n]$ and $j \in[n]$. Let $\mathbf{P}=\left(p_{i j}\right)$, and $\boldsymbol{\Gamma}$ its Hadamard (entry-wise) inverse, that is $\mathbf{P} \odot \boldsymbol{\Gamma}=\mathbf{1 1}^{\top}$, which is guaranteed to exist since $p_{i j}>0$ for all $i, j \in[n]$. When $\mathbb{E}[\mathbf{x}]=\mathbf{0}$, we will use

$$
\widehat{\mathbf{\Sigma}}=\frac{1}{N} \sum_{k=1}^{N} \mathbf{y}^{(k)} \mathbf{y}^{(k)^{\top}} \odot \boldsymbol{\Gamma} .
$$

Next we show that (3) is unbiased. We consider a slight generalization and assume the population mean is known and non-zero given by $\mathbb{E}[\mathbf{x}]=\boldsymbol{\mu}$. The estimator (3) becomes

$$
\widehat{\boldsymbol{\Sigma}}=\frac{1}{N} \sum_{k=1}^{N}\left(\mathbf{y}^{(k)}-\boldsymbol{\delta}^{(k)} \odot \boldsymbol{\mu}\right)\left(\mathbf{y}^{(k)}-\boldsymbol{\delta}^{(k)} \odot \boldsymbol{\mu}\right)^{\top} \odot \boldsymbol{\Gamma} .
$$

The centered variable satisfies $\mathbf{y}^{(k)}-\boldsymbol{\delta}^{(k)} \odot \boldsymbol{\mu}=\boldsymbol{\delta}^{(k)} \odot\left(\mathbf{x}^{(k)}-\right.$ $\boldsymbol{\mu})$, so we may write its expectation

$$
\begin{aligned}
& \mathbb{E}\left[\left(\mathbf{y}^{(k)}-\boldsymbol{\delta}^{(k)} \odot \boldsymbol{\mu}\right)\left(\mathbf{y}^{(k)}-\boldsymbol{\delta}^{(k)} \odot \boldsymbol{\mu}\right)^{\top}\right] \\
& =\mathbb{E}\left[\left(\boldsymbol{\delta}^{(k)} \odot\left(\mathbf{x}^{(k)}-\boldsymbol{\mu}\right)\right)\left(\boldsymbol{\delta}^{(k)} \odot\left(\mathbf{x}^{(k)}-\boldsymbol{\mu}\right)\right)^{\top}\right]
\end{aligned}
$$

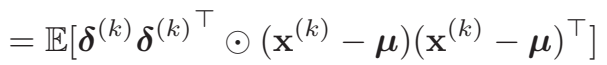

$$
\begin{aligned}
& =\mathbb{E}\left[\boldsymbol{\delta}^{(k)} \boldsymbol{\delta}^{(k)^{\top}}\right] \odot \mathbb{E}\left[\left(\mathbf{x}^{(k)}-\boldsymbol{\mu}\right)\left(\mathbf{x}^{(k)}-\boldsymbol{\mu}\right)^{\top}\right]=\mathbf{P} \odot \boldsymbol{\Sigma} .
\end{aligned}
$$

With this we can show that expectation of $\widehat{\Sigma}$ is $\boldsymbol{\Sigma}$, hence we have the following.

Proposition 1. (3) is an unbiased estimator for $\boldsymbol{\Sigma}$.

Our error bound for (3) is given in Theorem 1. Previously, [10] characterized the estimation error of (3) when $\delta$ has independent and identically distributed coordinates. Later, [14] derived error bounds for the non uniform dependent setting, however, as we will see in Section IV, their bounds do not match those of [10] or ours, and thus are sub-optimal.

b) Unbiased estimator under MCAR observations with unknown observation probabilities: Here we consider a more realistic scenario in which the observation probabilities are unknown and have to be estimated from data. For this we define the matrix of empirical observation probability

$$
\widehat{\mathbf{P}}=\frac{1}{N} \sum_{k=1}^{N} \boldsymbol{\delta}^{(k)} \boldsymbol{\delta}^{(k)^{\top}},
$$

and denote its entries by $\hat{p}_{i j}$. The quantity $\hat{p}_{i j}$ is equal to zero when the $i$-th and $j$-th variables are not observed together. Since we can only estimate the entries of the covariance matrix for which there are observations, we define the set $\mathcal{E}=\{(i, j)$ : $\left.\hat{p}_{i j} \neq 0\right\}$. Let $\boldsymbol{\Sigma}_{\mathcal{E}}$ be matrix whose entries in $\mathcal{E}$ coincide with the population covariance, and are zero otherwise. Let $\widehat{\boldsymbol{\Gamma}}=\left(\hat{\gamma}_{i j}\right)$ be the Hadamard inverse of $\max \left(\widehat{\mathbf{P}}, \frac{1}{N} \mathbf{1 1}^{\top}\right)$, which satisfies $\hat{\gamma}_{i j} \hat{p}_{i j}=1$ if $(i, j) \in \mathcal{E}$, and $\hat{\gamma}_{i j} \hat{p}_{i j}=0$ otherwise. An unbiased estimator for $\Sigma_{\mathcal{E}}$ is given by

$$
\widehat{\boldsymbol{\Sigma}}=\frac{1}{N} \sum_{k=1}^{N} \mathbf{y}^{(k)} \mathbf{y}^{(k)^{\top}} \odot \widehat{\boldsymbol{\Gamma}} .
$$


In fact, we can use the more general estimator

$$
\widehat{\boldsymbol{\Sigma}}=\frac{1}{N} \sum_{k=1}^{N}\left(\mathbf{y}^{(k)}-\boldsymbol{\delta}^{(k)} \odot \boldsymbol{\mu}\right)\left(\mathbf{y}^{(k)}-\boldsymbol{\delta}^{(k)} \odot \boldsymbol{\mu}\right)^{\top} \odot \widehat{\boldsymbol{\Gamma}}
$$

when the population mean $\boldsymbol{\mu}$ is non zero. To see that this estimator is unbiased, we write each term in the sum as $\left(\mathbf{x}^{(k)}-\boldsymbol{\mu}\right)\left(\mathbf{x}^{(k)}-\boldsymbol{\mu}\right)^{\top} \odot \boldsymbol{\delta}^{(k)} \boldsymbol{\delta}^{(k)^{\top}} \odot \widehat{\boldsymbol{\Gamma}}$. Applying conditional expectation, and independence of $\boldsymbol{\Delta}$ and $\mathrm{X}$ (MCAR assumption) we get

$$
\begin{aligned}
& \mathbb{E}\left[\left(\mathbf{x}^{(k)}-\boldsymbol{\mu}\right)\left(\mathbf{x}^{(k)}-\boldsymbol{\mu}\right)^{\top} \odot \boldsymbol{\delta}^{(k)} \boldsymbol{\delta}^{(k)^{\top}} \odot \widehat{\boldsymbol{\Gamma}} \mid \boldsymbol{\Delta}\right] \\
& =\mathbb{E}\left[\left(\mathbf{x}^{(k)}-\boldsymbol{\mu}\right)\left(\mathbf{x}^{(k)}-\boldsymbol{\mu}\right)^{\top} \mid \boldsymbol{\Delta}\right] \odot \boldsymbol{\delta}^{(k)} \boldsymbol{\delta}^{(k)^{\top}} \odot \widehat{\boldsymbol{\Gamma}} \\
& =\boldsymbol{\Sigma} \odot \boldsymbol{\delta}^{(k)} \boldsymbol{\delta}^{(k)^{\top}} \odot \widehat{\boldsymbol{\Gamma}} .
\end{aligned}
$$

Adding up all terms produces

$\mathbb{E}[\widehat{\boldsymbol{\Sigma}} \mid \boldsymbol{\Delta}]=\frac{1}{N} \sum_{k=1}^{N} \boldsymbol{\Sigma} \odot \boldsymbol{\delta}^{(k)} \boldsymbol{\delta}^{(k)^{\top}} \odot \widehat{\boldsymbol{\Gamma}}=\boldsymbol{\Sigma} \odot \widehat{\mathbf{P}} \odot \widehat{\boldsymbol{\Gamma}}=\boldsymbol{\Sigma}_{\mathcal{E}}$ If all $\hat{p}_{i j}>0$, then $\widehat{\mathbf{P}} \odot \widehat{\boldsymbol{\Gamma}}=\mathbf{1 1}{ }^{\top}, \boldsymbol{\Sigma}_{\mathcal{E}}=\boldsymbol{\Sigma}$ and $\mathbb{E}[\widehat{\boldsymbol{\Sigma}}]=\boldsymbol{\Sigma}$.

Proposition 2. Conditioned on $\boldsymbol{\Delta}$, (5) is an unbiased estimator for $\boldsymbol{\Sigma}_{\mathcal{E}}$. If all $\hat{p}_{i j}>0$, then (5) is unbiased for $\boldsymbol{\Sigma}$.

A similar but biased estimator that does not know the missing data distribution was used as part of the banded and sparse covariance estimators from [13], and the inverse covariance estimator from [16]. However, to the best of our knowledge we are the first to establish estimation error bounds for an estimator of this form in Theorem 2.

c) Unbiased estimator under CMCAR observations: The CMCAR mechanism presented here generalizes the MCAR to allow missing data with a time varying distribution. One instance of this mechanism are active sequential observations, where the observation probabilities depend on previously observed data. Another, is a MCAR mechanism with time varying observation probabilities. To the best of our knowledge, this type of missing data mechanism has not been considered for covariance matrix estimation.

The index $t=1,2, \cdots$ denotes time (or iteration number). The observed vectors at time $t$ are $\left\{\mathbf{y}^{(t, 1)}, \cdots, \mathbf{y}^{\left(t, N_{t}\right)}\right\}$, which can be stored as columns of the $n \times N_{t}$ dimesional matrix $\mathbf{Y}_{t}$. Each of these vectors is defined as $\mathbf{y}^{(t, k)}=\boldsymbol{\delta}^{(t, k)} \odot \mathbf{x}^{(t, k)}$, where $\left\{\boldsymbol{\delta}^{(t, k)}\right\}_{k=1}^{N_{t}}$ are identically distributed Bernoulli with sampling probabilities $p_{i j}^{(t)}$. We assume that for $t \geq 1$,

1) Complete data is i.i.d.. For $k \in\left[N_{t}\right]$, and $t \in[T]$, the complete observations $\mathbf{x}^{(t, k)}$ are i.i.d. copies of $\mathbf{x}$.

2) Stochastic observation parameters. The sampling probabilities are a function of previous observations, thus $p_{i j}^{(t)}=f_{i j t}\left(\mathbf{Y}_{t-1}, \mathbf{Y}_{t-2}, \cdots, \mathbf{Y}_{1}\right)$.

3) Conditionally i.i.d. observations. Conditioned on the event $\left\{\mathbf{Y}_{\tau}: \tau<t\right\}$, the random vectors $\mathbf{y}^{(t, 1)}, \cdots, \mathbf{y}^{\left(t, N_{t}\right)}$ are i.i.d..

4) Conditionally MCAR (CMCAR) mechanism. Conditioned on the event $\left\{\mathbf{Y}_{\tau}: \tau<t\right\}$, the vectors $\left\{\boldsymbol{\delta}^{(t, k)}\right\}_{k=1}^{N_{t}}$ are i.i.d., and independent of $\mathbf{x}^{(t, k)}$.

The sampling probabilities are positive (i.e., $p_{i j}^{(t)}>0$ for all $i, j, t)$. We will also use the matrices $\mathbf{P}_{t}=\left(p_{i j}^{(t)}\right)$, and $\boldsymbol{\Gamma}_{t}$, which are Hadamard inverses, that is, $\mathbf{P}_{t} \odot \boldsymbol{\Gamma}_{t}=\mathbf{1 1 ^ { \top }}$. For simplicity, conditional expectation is abbreviated as

$$
\mathbb{E}_{t-1}[\cdot]=\mathbb{E}\left[\cdot \mid \mathbf{Y}_{\tau}: \tau<t\right]
$$

Below we present some useful quantities

$$
\begin{aligned}
\mathbf{Z}_{t} & =\mathbf{Y}_{t} \mathbf{Y}_{t}^{\top} \odot \boldsymbol{\Gamma}_{t} \\
\mathbf{W}_{T} & =\sum_{t=1}^{T}\left(\mathbf{Z}_{t}-N_{t} \boldsymbol{\Sigma}\right) \\
\widehat{\boldsymbol{\Sigma}}_{T} & =\frac{\sum_{t=1}^{T} \mathbf{Z}_{t}}{\sum_{t=1}^{T} N_{t}}
\end{aligned}
$$

Some facts about $\mathbf{Z}_{t}, \mathbf{W}_{t}$ and $\widehat{\boldsymbol{\Sigma}}_{T}$ are stated below.

1) $\mathbf{Z}_{t}$ is an unbiased estimator for $N_{t} \boldsymbol{\Sigma}$. This follows from an application of conditional expectation, and using (3),

$$
\begin{aligned}
\mathbb{E}_{t-1}\left[\mathbf{Z}_{t}\right] & =\mathbb{E}_{t-1}\left[\mathbf{Y}_{t} \mathbf{Y}_{t}^{\top} \odot \boldsymbol{\Gamma}_{t}\right] \\
& =\mathbb{E}_{t-1}\left[\mathbf{Y}_{t} \mathbf{Y}_{t}^{\top}\right] \odot \boldsymbol{\Gamma}_{t} \\
& =N_{t} \boldsymbol{\Sigma} \odot \mathbf{P}_{t} \odot \boldsymbol{\Gamma}_{t} \\
& =N_{t} \boldsymbol{\Sigma} .
\end{aligned}
$$

2) $\mathbf{W}_{T}$ is a zero mean matrix martingale. The martingale property states that $\mathbb{E}_{T}\left[\mathbf{W}_{T+1}\right]=\mathbf{W}_{T}$. We can verify this applying conditional expectation,

$$
\mathbb{E}_{T}\left[\mathbf{W}_{T+1}\right]=\mathbb{E}_{T}\left[\mathbf{Z}_{T+1}-N_{T+1} \boldsymbol{\Sigma}\right]+\mathbb{E}_{T}\left[\mathbf{W}_{T}\right]=\mathbf{W}_{T} .
$$

This property implies that $\mathbb{E}\left[\mathbf{W}_{T}\right]=\mathbb{E}\left[\mathbf{W}_{1}\right]=\mathbf{0}$.

3) $\widehat{\boldsymbol{\Sigma}}_{T}$ is an unbiased estimator for $\boldsymbol{\Sigma}$. This is a direct consequence of property 2) and the identity $\mathbf{W}_{T}=$ $\left(\widehat{\boldsymbol{\Sigma}}_{T}-\boldsymbol{\Sigma}\right)\left(\sum_{t=1}^{T} N_{t}\right)$.

Performance of this estimator is presented in Theorem 3. Given Propositions 1 and 2 , the observations $\mathbf{y}^{(k)}$ can always be centered to have mean zero, provided the population mean is known. For the rest of the paper we will assume, without loss of generality, that the mean is known and $\boldsymbol{\mu}=\mathbf{0}$.

\section{Unknown mean}

When the mean is unknown, centering using an estimate for the population mean is not sufficient to construct an unbiased estimator. To see this, consider

$$
\frac{1}{N} \sum_{k=1}^{N}\left(\mathbf{y}^{(k)}-\boldsymbol{\delta}^{(k)} \odot \overline{\mathbf{y}}\right)\left(\mathbf{y}^{(k)}-\boldsymbol{\delta}^{(k)} \odot \overline{\mathbf{y}}\right)^{\top} \odot \widehat{\boldsymbol{\Gamma}},
$$

which was utilized in [13], [16] when the mean and missing data distributions are unknown. Although (9) is a consistent estimator for $\Sigma$ [13, Lemma 2.1], it is also biased. To see the latter, notice that in the complete data case, (9) becomes $(1 / N) \sum_{k=1}^{N}\left(\mathbf{x}^{(k)}-\overline{\mathbf{x}}\right)\left(\mathbf{x}^{(k)}-\overline{\mathbf{x}}\right)^{\top}$, which has expectation equal to $(N-1 / N) \boldsymbol{\Sigma}$. A similar situation is encountered when $\mathbf{P}$ is known. In appendix A, we discuss unbiased alternatives to (9), for known and unknown missing data distribution. However, the expressions are much more cumbersome, thus a theoretical error study is left for future work. 


\section{ERROR BOUNDS}

We start this section by introducing some concepts related to sub-Gaussian vectors and notions of complexity of covariance matrices. In Theorems 1, 2, and 3 we present error bounds in operator norm for each estimator. All results of this section depend on new quantities we denote scaled effective rank, which generalize the effective rank to account for partial observations. All our bounds can be viewed as non trivial applications of a moment inequality found in [49]. After some simplifications, our results are summarized in Table I. Based on Propositions 1 and 2, in this section we assume the population mean is known and equal to zero.

\section{A. Preliminaries and assumptions}

We first introduce a proxy for the rank of a matrix.

Definition 1. The effective rank [10], [50] of a $n \times n$ matrix A is defined as

$$
r(\mathbf{A})=\frac{\|\mathbf{A}\|_{\star}}{\|\mathbf{A}\|} .
$$

The effective rank obeys $1 \leq \mathrm{r}(\mathbf{A}) \leq \operatorname{rank}(\mathbf{A}) \leq n$. When A is positive semi-definite, $\|\mathbf{A}\|_{\star}=\operatorname{tr}(\mathbf{A})$. The effective rank, as we will see in latter sections, is the parameter that quantifies the sample complexity of covariance estimation.

We introduce sub-Gaussian variables and vectors below.

Definition 2 ([50]). If $\mathbb{E}\left[\exp \left(z^{2} / K^{2}\right)\right] \leq 2$ holds for some $K>0$, we say $z$ is sub-Gaussian. If $\mathbb{E}[\exp (|z| / K)] \leq 2$ holds for some $K>0$, we say $z$ is sub-exponential.

Definition 3 ([50]). For a real valued random variable $z$, the sub-Gaussian and sub-exponential norms are defined as

$$
\|z\|_{\Psi_{\alpha}}=\inf \left\{u>0: \mathbb{E}\left[\exp \left(|z|^{\alpha} / u^{\alpha}\right)\right] \leq 2\right\}
$$

for $\alpha=2$ and $\alpha=1$ respectively.

Definition 4. If a random vector $\mathbf{z}$ in $\mathbb{R}^{n}$ satisfies

$$
\|\mathbf{z}\|_{\Psi_{\alpha}}=\sup _{\mathbf{u}:\|\mathbf{u}\|=1}\left\|\mathbf{u}^{\top} \mathbf{z}\right\|_{\Psi_{\alpha}}<\infty
$$

it is called sub-Gaussian or sub-exponential, for $\alpha=2$ or $\alpha=1$ respectively.

We will work under Assumption 1 detailed below, which is standard in the covariance estimation literature [11], [10].

Assumption 1. The distribution of $\mathrm{x}$ obeys.

- $\mathbf{x}$ is a sub-Gaussian vector, therefore there is a constant c so that $\mathbb{E}\left[\left|\mathbf{u}^{\top} \mathbf{x}\right|^{r}\right]^{1 / r} \leq c\left\|\mathbf{u}^{\top} \mathbf{x}\right\|_{\Psi_{2}} \sqrt{r}$ for every unit vector $\mathbf{u} \in \mathbb{R}^{n}$, and $r \geq 1$.

- There is a constant $c_{0}$ so that for all $\mathbf{u} \in \mathbb{R}^{n}$, then $\left\|\mathbf{u}^{\top} \mathbf{x}\right\|_{\Psi_{2}}^{2} c_{0} \leq \mathbb{E}\left(\mathbf{u}^{\top} \mathbf{x}\right)^{2}$

Finally we introduce a generalization of the effective rank.

Definition 5. A scaled effective rank of a matrix $\boldsymbol{\Sigma}$, is any quantity that takes values in $[\mathrm{r}(\boldsymbol{\Sigma}), \mathrm{r}(\boldsymbol{\Sigma}) / \rho]$ for some constant $0<\rho<1$, where the constant does not depend on $\Sigma$.
Examples that will appear in this section are

$$
\begin{aligned}
& \mathrm{r}_{\min }(\boldsymbol{\Sigma}, \mathbf{P})=\frac{1}{\|\boldsymbol{\Sigma}\|} \max _{j \in[n]} \sum_{i=1}^{n} \frac{\Sigma_{i i}}{p_{i j}}=\frac{1}{\|\boldsymbol{\Sigma}\|}\|\operatorname{diag}(\boldsymbol{\Sigma}) \boldsymbol{\Gamma}\|_{1 \rightarrow 1}, \\
& \mathrm{r}_{2}(\boldsymbol{\Sigma}, \mathbf{P})=\frac{1}{\|\boldsymbol{\Sigma}\|}\left\|\operatorname{diag}(\boldsymbol{\Sigma})^{1 / 2} \boldsymbol{\Gamma} \operatorname{diag}(\boldsymbol{\Sigma})^{1 / 2}\right\|_{F}, \\
& \tilde{\mathrm{r}}_{\min }(\boldsymbol{\Sigma}, \mathbf{p})=\frac{1}{\|\boldsymbol{\Sigma}\|} \frac{1}{\min _{i} p_{i i}} \sum_{i=1}^{n} \frac{\Sigma_{i i}}{p_{i i}}, \\
& \tilde{\mathrm{r}}_{2}(\boldsymbol{\Sigma}, \mathbf{p})=\frac{1}{\|\boldsymbol{\Sigma}\|} \sum_{i=1}^{n} \frac{\Sigma_{i i}}{p_{i i}^{2}},
\end{aligned}
$$

where $\mathbf{p}$ is a vector containing the diagonal entries of $\mathbf{P}$.

\section{B. MCAR case with known distribution}

Now we state our main result for the MCAR model.

Theorem 1. Suppose the random vector $\mathrm{x}$ obeys Assumption 1. If all $p_{i j}>0$, and also $N \geq e^{2}$ and $n \geq 3$, we have that (3) satisfies

$$
\begin{aligned}
& \mathbb{E}\left[\|\widehat{\boldsymbol{\Sigma}}-\boldsymbol{\Sigma}\|^{2}\right]^{1 / 2} \leq C\|\boldsymbol{\Sigma}\| \\
& \left(\sqrt{\frac{8 e \log (n) \mathrm{r}_{\min }(\boldsymbol{\Sigma}, \mathbf{P})}{N}}+\frac{4 e^{2} \log (n) \log (N) \mathrm{r}_{2}(\boldsymbol{\Sigma}, \mathbf{P})}{N}\right),
\end{aligned}
$$

where $C$ is an universal constant. In addition, if $\boldsymbol{\delta}$ has independent entries, the parameters $\mathrm{r}_{\min }(\boldsymbol{\Sigma}, \mathbf{P})$ and $\mathrm{r}_{2}(\boldsymbol{\Sigma}, \mathbf{P})$ in (10) can be replaced by $\tilde{\mathrm{r}}_{\min }(\boldsymbol{\Sigma}, \mathbf{p})$ and $\tilde{\mathrm{r}}_{2}(\boldsymbol{\Sigma}, \mathbf{p})$, respectively.

The upper bound from Theorem 1 suggests that if variable $x_{i}$ has high variance (high $\Sigma_{i i}$ ) or if it is strongly correlated with other variables (high $\Sigma_{i j}$ ), then the effect of missing data can be more severe, since the upper bound increases with $\Sigma_{i i} / p_{i}$ and $\Sigma_{i j} / p_{i j}$.

Theorem 1 is an improvement over previous bounds under the MCAR mechanism with known missing data distribution. In particular, we obtain better convergence rates than [14], and consider a more general missing data mechanism than [10]. However, these previous studies obtained high probability error bounds, while here we bound the expectation.

a) Comparison with covariance estimation with complete observations: In this case, $p_{i i}=1$ for all $i \in[n]$, which implies that all $p_{i j}=1$, and the scaled effective rank coincides with the effective rank. Several recent papers [10], [11], [12] have obtained bounds in operator norm that scale as

$$
\mathcal{O}\left(\sqrt{\frac{\mathrm{r}(\boldsymbol{\Sigma})}{N}}+\frac{\mathrm{r}(\boldsymbol{\Sigma})}{N}\right)=\mathcal{O}\left(\sqrt{\frac{\mathrm{r}(\boldsymbol{\Sigma})}{N}} \vee \frac{\mathrm{r}(\boldsymbol{\Sigma})}{N}\right),
$$

where the $\mathcal{O}$ notation hides constants and logarithmic dependencies on $n$ and $N$. Our bound in spectral norm matches those of [10], [11] up to $\log (N)$ factors. For the Gaussian case, it was shown in [12] that dimension free bounds can be attained (without any log terms). It is also worth mentioning that the error bounds from [10], [11] are high probability results based on variations of matrix Bernstein inequalities for the operator norm. In [10], one is used for sums of bounded random matrices, while [11] applies a version for unbounded matrices. Our results are based in a moment inequality for 
sums of independent, possibly unbounded matrices from [49], thus significantly simplifying the proofs.

b) Sample complexity: We can guarantee covariance estimation error in operator norm of magnitude

$$
\mathbb{E}\left[\|\widehat{\boldsymbol{\Sigma}}-\boldsymbol{\Sigma}\|^{2}\right]^{1 / 2} \leq \epsilon\|\boldsymbol{\Sigma}\|
$$

if the number of observations obeys

$$
N \geq 8 e \log (n) \mathrm{r}_{\min }(\boldsymbol{\Sigma}, \mathbf{P})\left(e \rho \log (N) \vee \frac{2 C_{2}}{\epsilon}\right)^{2},
$$

where $\rho=\mathrm{r}_{2}(\boldsymbol{\Sigma}, \mathbf{P}) / \mathrm{r}_{\min }(\boldsymbol{\Sigma}, \mathbf{P})$. Compared with the case of complete observations, the missing data case requires extra samples by a factor of $\mathrm{r}_{\min }(\boldsymbol{\Sigma}, \mathbf{P}) / \mathrm{r}(\boldsymbol{\Sigma})$ to attain equal accuracy. When $\delta$ has independent coordinates, a simpler sufficient condition is given by

$$
N \geq 8 e \log (n) \tilde{\mathrm{r}}_{\min }(\boldsymbol{\Sigma}, \mathbf{p})\left(e \log (N) \vee \frac{2 C_{2}}{\epsilon}\right)^{2} .
$$

This condition can be derived from the looser bound that depends on $\tilde{\mathrm{r}}_{2}(\boldsymbol{\Sigma}, \mathbf{p})$ and $\tilde{\mathrm{r}}_{\min }(\boldsymbol{\Sigma}, \mathbf{p})$. In this case, both $\tilde{\mathrm{r}}_{2}(\boldsymbol{\Sigma}, \mathbf{p})$ and $\mathrm{r}_{\min }(\boldsymbol{\Sigma}, \mathbf{p})$ are upper bounded by $\tilde{\mathrm{r}}_{\min }(\boldsymbol{\Sigma}, \mathbf{p})$.

c) Parameter scaling: Each entry of the sample covariance matrix is a (weighted) average of a different number of observations. In particular the $i j$ off-diagonal element of $\widehat{\boldsymbol{\Sigma}}$ is observed $N p_{i j}$ times on average. This reduced data acquisition rate is reflected in an increased error bound of the same order.

d) Comparison with [10] and [14]: Since the bounds from [10] assume uniform independent observation variables, we will compare under that setting. Lounici [10] establishes that with probability at least $1-e^{-\nu}$

$$
\begin{aligned}
& \|\widehat{\boldsymbol{\Sigma}}-\boldsymbol{\Sigma}\| \leq C\|\boldsymbol{\Sigma}\| \\
& \max \left\{\sqrt{\frac{\mathrm{r}(\boldsymbol{\Sigma})(\nu+\log (2 n))}{p^{2} N}},\right. \\
& \left.\frac{\mathrm{r}(\boldsymbol{\Sigma})(\nu+\log (2 n))\left(C_{1} p+\nu+\log (N)\right)}{p^{2} N}\right\},
\end{aligned}
$$

where $C, C_{1}>0$ are universal constants. On the other hand, Park and Lim [14] derived a bound under MCAR observations, which after simplification under the uniform and independent assumption is reduced to

$$
\begin{aligned}
& \|\widehat{\boldsymbol{\Sigma}}-\boldsymbol{\Sigma}\| \leq C\|\boldsymbol{\Sigma}\| n \\
& \max \left\{\sqrt{\frac{\mathrm{r}(\boldsymbol{\Sigma})(\nu+\log (2 n))}{p^{7 / 2} N}},\right. \\
& \left.\frac{\mathrm{r}(\boldsymbol{\Sigma})(\nu+\log (2 n))\left(C_{1} p+\nu+\log (N)\right)}{p^{2} N}\right\},
\end{aligned}
$$

also with probability at least $1-e^{\nu}$. Setting $\nu=\log (2 n)$, and for large enough $N,(12)$ and Theorem 1 imply that the estimation error scales as

$$
\mathcal{O}\left(\sqrt{\frac{\mathrm{r}(\boldsymbol{\Sigma}) \log (2 n)}{p^{2} N}}\right)
$$

while the bound (13) has a worst rate by a factor of $n / p^{3 / 4}$. We can also compare in terms of sample complexity. Lounici's bound implies the following sample complexity condition

$$
N \geq C \frac{\mathrm{r}(\boldsymbol{\Sigma})}{p^{2}} \log (2 n)\left(\frac{1}{\epsilon} \vee\left(\log (n)+\log (N)+c_{1} p\right)\right)^{2} .
$$

The bound from [14] implies the condition

$$
N \geq C \frac{\mathrm{r}(\boldsymbol{\Sigma})}{p^{1 / 2}} \log (2 n)\left(\frac{n}{p^{3 / 2} \epsilon} \vee\left(\log (n)+\log (N)+c_{1} p\right)\right)^{2}
$$

which requires a significant larger number of samples. This can be observed by the additional $n^{2}$ factor, and an asymptotic dependency on the missing data in the order of $1 / p^{7 / 2}$. [14] points out the necessity for an improved rate of convergence, which is partially addressed by Theorem 1 , however since our result is an expectation bound, more research is needed to obtain concentration inequalities. See Table I for a summary of the sample complexity comparison from this section.

\section{MCAR case with unknown distribution}

The main disadvantage of (3) and Theorem 1 is that in practice, the distribution of $\delta$ is unknown. Here we present a our bound for (5), which depends on $\widehat{\mathbf{P}}$ instead of $\mathbf{P}$.

Theorem 2. Suppose the random vector $\mathbf{x}$ satisfies Assumption 1 , with $N \geq e^{2}$ and $n \geq 3$. Also assume that for all $i \in[n]$ there is a $k \in[N]$ so that $\delta_{i}^{(k)}=1$. Conditioned on the missing data pattern $\boldsymbol{\Delta}$, the estimator from (5) satisfies

$$
\begin{aligned}
& \mathbb{E}\left[\left\|\widehat{\boldsymbol{\Sigma}}-\boldsymbol{\Sigma}_{\mathcal{E}}\right\|^{2} \mid \boldsymbol{\Delta}\right]^{1 / 2} \leq C\|\boldsymbol{\Sigma}\| \\
& \left(\sqrt{\frac{8 e \log (n) \mathrm{r}_{\min }(\boldsymbol{\Sigma}, \widehat{\mathbf{P}})}{N}}+\frac{4 e^{2} \log (n) \log (N) \mathrm{r}_{2}(\boldsymbol{\Sigma}, \widehat{\mathbf{P}})}{N}\right)
\end{aligned}
$$

where $C$ is a universal numerical constant, $\boldsymbol{\Sigma}_{\mathcal{E}}=\mathbb{E}[\widehat{\boldsymbol{\Sigma}}]$, and

$$
\begin{aligned}
\mathrm{r}_{\min }(\boldsymbol{\Sigma}, \widehat{\mathbf{P}}) & =\frac{1}{\|\boldsymbol{\Sigma}\|} \max _{j} \sum_{i:(i, j) \in \mathcal{E}} \frac{\Sigma_{i i}}{\widehat{p}_{i j}} \\
\mathrm{r}_{2}(\boldsymbol{\Sigma}, \widehat{\mathbf{P}}) & =\frac{1}{\|\boldsymbol{\Sigma}\|} \sqrt{\sum_{(i, j) \in \mathcal{E}} \frac{\Sigma_{i i} \Sigma_{j j}}{\widehat{p}_{i j}^{2}}} .
\end{aligned}
$$

In general, (14) corresponds to an inequality between random variables that are functions of $\boldsymbol{\Delta}$, however Theorem 2 is also valid if $\Delta$ is a deterministic matrix. The parameters from Theorem 2 are scaled effective ranks, since they obey

$$
\begin{array}{r}
\mathrm{r}(\boldsymbol{\Sigma}) \leq \mathrm{r}_{\min }(\boldsymbol{\Sigma}, \widehat{\mathbf{P}}) \leq \frac{\mathrm{r}(\boldsymbol{\Sigma})}{\widehat{p}_{\min }} \\
\mathrm{r}(\boldsymbol{\Sigma}) \leq \mathrm{r}_{2}(\boldsymbol{\Sigma}, \widehat{\mathbf{P}}) \leq \frac{\mathrm{r}(\boldsymbol{\Sigma})}{\widehat{p}_{\min }}
\end{array}
$$

almost surely, where $\widehat{p}_{\min }=\min _{(i, j) \in \mathcal{E}} \widehat{p}_{i j}$. 
a) Comparison with Theorem 1: The proposed estimators, (3) and (5), can correct for the bias introduced to the sample covariance matrix due to missing observations, by normalizing its entries with the matrices $\Gamma$ and $\widehat{\Gamma}$, respectively. The bound from Theorem 1 is characterized by scaled effective rank parameters that depend on the observation probability matrix P. In Theorem 2 we obtained the same bound, however now the scaled effective rank parameters have replaced the observation probability matrix with its estimate $\widehat{\mathbf{P}}$. Theorems 1 and 2 suggest that, asymptotically (large $N$ ), both estimators have the same performance, since $\widehat{\mathbf{P}}$ converges to $\mathbf{P}$. As discussed in Section III, for $\widehat{\boldsymbol{\Sigma}}$ to be unbiased, we require that all $\widehat{p}_{i j}>0$, meaning that all pairs of variables must be observed together at least once. Its counterpart in Theorem 1 is the condition that all $p_{i j}>0$.

b) Comparison with [13]: Let us denote the estimator (9) by $\tilde{\Sigma}$, Lemma 2.1 in [13] implies that with probability at least $1-e^{-\nu}, \tilde{\boldsymbol{\Sigma}}$ obeys

$$
\|\tilde{\boldsymbol{\Sigma}}-\boldsymbol{\Sigma}\| \leq C\|\boldsymbol{\Sigma}\|\left(\sqrt{\frac{c_{1}+c_{2} n+\nu}{N \hat{p}_{\min }}}+\frac{c_{1}+c_{2} n+\nu}{N \hat{p}_{\min }}\right) .
$$

Essentially, the dominating parameter in the above bound is the quantity $n / \hat{p}_{\min }$. First, this bound depends on the least observed entry (through $\hat{p}_{\text {min }}$ ), and second, it depends on $n$ instead of the effective rank, which always satisfies $\mathrm{r}(\boldsymbol{\Sigma}) \leq n$. Therefore, this bound is always larger than the right hand side of (15) and (16).

c) A closer look at (3) and (5): for (3), the estimation error of the $i j$ entry is

$$
\mathbb{E}\left[\left|\widehat{\boldsymbol{\Sigma}}_{i j}-\boldsymbol{\Sigma}_{i j}\right|^{2}\right]=\frac{\operatorname{var}\left[\left(x_{i} x_{j}\right)^{2}\right]+\left(1-p_{i j}\right) \boldsymbol{\Sigma}_{i j}^{2}}{p_{i j} N} .
$$

A similar calculation produces an entry-wise error for (5) equal to

$$
\mathbb{E}\left[\left|\widehat{\boldsymbol{\Sigma}}_{i j}-\boldsymbol{\Sigma}_{i j}\right|^{2}\right]=\mathbb{E}\left[\frac{1}{\hat{p}_{i j}}\right] \frac{\operatorname{var}\left[\left(x_{i} x_{j}\right)^{2}\right]}{N} .
$$

For complete data $p_{i j}=\hat{p_{i j}}=1$, both estimators have the same error. When there is missing data, if $N$ is large, $\mathbb{E}\left[1 / \hat{p}_{i j}\right]$ converges to $1 / p_{i j}$, and thus (3) will always be outperformed by (5). Note that since $\mathbb{E}\left[x_{i} x_{j}\right]^{2}=\Sigma_{i j}^{2}$, (18) is composed of a variance term, while (17) has a variance and an additional bias. This occurs because the $i j$ entries of both estimators are a sum of $\hat{p}_{i j} N$ terms, however since for (3), the number of terms does not coincide with the dividing factor $p_{i j} N$, each term is not an exact average, unlike the case of (5).

\section{CMCAR case with known distribution}

Now we present an error bound for (7).

Theorem 3. Suppose $\mathbf{P}^{(t+1)}=f_{t}\left(\widehat{\boldsymbol{\Sigma}}_{t}\right)$ for all $t \geq 1$ where $f_{t}(\cdot)$ are bounded, in the sense that there is a constant $\alpha$ so that for all $i$ and for all $t, p_{i j}^{(t)} \geq \alpha>0$ almost surely. Under the same assumptions of Theorem 1, (7) obeys

$$
\begin{aligned}
\mathbb{E}\left[\left\|\widehat{\boldsymbol{\Sigma}}_{T}-\boldsymbol{\Sigma}\right\|^{2}\right]^{1 / 2} & \leq \tilde{C}\|\boldsymbol{\Sigma}\| \sqrt{\log (n+1)} \\
& \times\left[\sqrt{\frac{8 e \log (n)}{N} \varepsilon_{1}}+\frac{4 e^{2} \log (n) L}{N} \varepsilon_{2}\right],
\end{aligned}
$$

where $\tilde{C}$ is an universal constant, $\varepsilon_{1}, \varepsilon_{2}$ are given by

$$
\begin{aligned}
& \varepsilon_{1}\left(\boldsymbol{\Sigma},\left\{\mathbf{P}^{(t)}, N_{t}\right\}_{t=1}^{T}\right)=\mathbb{E}\left[\frac{1}{N} \sum_{t=1}^{T} \mathrm{r}_{\min }\left(\boldsymbol{\Sigma}, \mathbf{P}^{(t)}\right) N_{t}\right] \\
& \varepsilon_{2}^{2}\left(\boldsymbol{\Sigma},\left\{\mathbf{P}^{(t)}, N_{t}\right\}_{t=1}^{T}\right)=\mathbb{E}\left[\frac{1}{L^{2}} \sum_{t=1}^{T} \mathrm{r}_{2}^{2}\left(\boldsymbol{\Sigma}, \mathbf{P}^{(t)}\right) \log ^{2}\left(N_{t}\right)\right]
\end{aligned}
$$

and $N=\sum_{t=1}^{T} N_{t}$, and $L^{2}=\sum_{t=1}^{T} \log ^{2}\left(N_{t}\right)$.

Note that when $T=1$, the bounds from Theorems 1 and 3 only differ on the $\sqrt{\log (n+1)}$ term and a constant. The estimation error depends on the behavior of the quantities $\varepsilon_{i}$, which depends on the observations, the missing data mechanism and $\left\{f_{t}\right\}_{t=1}^{T}$. In particular, when $f_{t}$ depends only on the index $t$, and not on the data, we have the case of a time varying MCAR mechanism. The assumptions of Theorem 3 guarantee that $\varepsilon_{i}$ are scaled effective ranks, thus

$$
\varepsilon_{i} \leq \frac{\mathrm{r}(\boldsymbol{\Sigma})}{\alpha}
$$

and $\widehat{\Sigma}_{T} \rightarrow \Sigma$ in probability. To establish that this bound is also consistent with Theorems 1 and 2 for $T>1$ we will analyze the limiting behavior of the expectations in the upper bound.

Proposition 3. Under the same assumptions of Theorem 3, if additionally $f_{t}=f$ is continuous, then

$$
\begin{aligned}
& \mathbf{P}^{(t)} \rightarrow \mathbf{P}^{\infty}, \\
& \mathrm{r}_{2}\left(\boldsymbol{\Sigma}, \mathbf{P}^{(t)}\right) \rightarrow \mathrm{r}_{2}\left(\boldsymbol{\Sigma}, \mathbf{P}^{\infty}\right), \\
& \mathrm{r}_{\min }\left(\boldsymbol{\Sigma}, \mathbf{P}^{(t)}\right) \rightarrow \mathrm{r}_{\min }\left(\boldsymbol{\Sigma}, \mathbf{P}^{\infty}\right),
\end{aligned}
$$

in probability, where $\mathbf{P}^{\infty}=f(\boldsymbol{\Sigma})$. Also we have convergence of the expectation, that is,

$$
\begin{array}{r}
\mathbb{E}\left[\mathrm{r}_{2}^{2}\left(\boldsymbol{\Sigma}, \mathbf{P}^{(t)}\right)\right] \rightarrow \mathrm{r}_{2}^{2}\left(\boldsymbol{\Sigma}, \mathbf{P}^{\infty}\right), \\
\mathbb{E}\left[\mathrm{r}_{\min }\left(\boldsymbol{\Sigma}, \mathbf{P}^{(t)}\right)\right] \rightarrow \mathrm{r}_{\min }\left(\boldsymbol{\Sigma}, \mathbf{P}^{\infty}\right) .
\end{array}
$$

Proof: Since $f$ is bounded and a continuous function of $\widehat{\boldsymbol{\Sigma}}_{t}$, so are $\mathrm{r}_{s} \circ f$, and $\mathrm{r}_{\min } \circ f$. All limits are implied by convergence in probability of $\widehat{\mathbf{\Sigma}}_{t}$ to its expected value, along with [51, Th. 2.3.4].

Proposition 3, and Theorems 1 and 3 imply that asymptotically, our estimators in (3) and (7) converge at the same rates. This is a direct consequence of the following limits, which are implied by Proposition 3

$$
\begin{aligned}
& \varepsilon_{1} \rightarrow \mathrm{r}_{\min }\left(\boldsymbol{\Sigma}, \mathbf{P}^{\infty}\right), \text { and } \\
& \varepsilon_{2} \rightarrow \mathrm{r}_{2}\left(\Sigma, \mathbf{P}^{\infty}\right) .
\end{aligned}
$$

We will finish the analysis of this estimator by establishing a lower bound on the sample complexity. 
Proposition 4. Suppose that $e<N_{t}$ for all $t \in[T]$, and the following conditions hold

$$
\begin{aligned}
& \mathbb{E}\left[\sum_{t=1}^{T} \mathrm{r}_{\min }\left(\boldsymbol{\Sigma}, \mathbf{P}^{(t)}\right) N_{t}\right] \\
& \geq \mathbb{E}\left[\sum_{t=1}^{T} 2 e^{3} \log (n) \log ^{2}\left(N_{t}\right) \mathrm{r}_{2}^{2}\left(\boldsymbol{\Sigma}, \mathbf{P}^{(t)}\right)\right], \\
N & \geq 32 e \tilde{C}^{2} \log (n) \log (n+1) \varepsilon_{1} \frac{1}{\epsilon^{2}}
\end{aligned}
$$

for some $T>1$ and some $\epsilon>0$, then

$$
\mathbb{E}\left[\left\|\widehat{\boldsymbol{\Sigma}}_{T}-\boldsymbol{\Sigma}\right\|^{2}\right]^{1 / 2} \leq \epsilon\|\boldsymbol{\Sigma}\|
$$

Proof: The first inequality implies

$$
\sqrt{\frac{8 e \log (n)}{N} \varepsilon_{1}} \geq \frac{2 e^{3} \log (n) L}{N} \varepsilon_{2} .
$$

Combining this with Theorem 3 leads to

$$
\mathbb{E}\left[\left\|\widehat{\boldsymbol{\Sigma}}_{T}-\boldsymbol{\Sigma}\right\|^{2}\right]^{1 / 2} \leq 2 \tilde{C}\|\boldsymbol{\Sigma}\|\left[\sqrt{\frac{8 e \log (n) \log (n+1)}{N} \varepsilon_{1}}\right] .
$$

Using the second assumption leads to the desired bound.

If we ignore logarithmic and constant factors the condition (27) would be in the same order of magnitude as the sample complexity we established for the MCAR estimators. Note that in this case there is an additional $\log (n+1)$ factor. The inequality (26) is a condition on the sequence $\left\{N_{t}\right\}$, and although it looks convoluted, it is easily satisfied if $T$ is large enough, and

$$
N_{t} / \log ^{2}\left(N_{t}\right)=\Omega\left(\log (n) \mathrm{r}_{2}^{2}\left(\boldsymbol{\Sigma}, \mathbf{P}^{\infty}\right) / \mathrm{r}_{\min }\left(\boldsymbol{\Sigma}, \mathbf{P}^{\infty}\right)\right) .
$$

This can be achieved for example if $N_{t}$ is an increasing sequence with $\lim _{t \rightarrow \infty} N_{t}=\infty$.

\section{EXPERIMENTS}

\section{A. Covariance estimation under MCAR observations}

1) Uniform MCAR observations: We first compare the estimators from (3) and (5), designed to handle MCAR observations with known and unknown missing data distribution respectively. The experimental setup is as follows.

- Data follows a Gaussian distribution with mean zero, and population covariance matrix $\boldsymbol{\Sigma}$. The eigenvalues are $\rho^{i}$ for $i=1, \cdots, n$, with $0<\rho<1$ chosen so that $\mathrm{r}(\boldsymbol{\Sigma})=$ 4. The eigenvectors are generated by applying the GramSchmidt method to a $n \times n$ random matrix with $\mathcal{N}(0,1)$ independent entries.

- The dimension is set to $n=50$. The MCAR missing data mechanism is uniform with independend entries and observation probabilities $p \in\{0.4,0.6,0.8\}$.

- We generate complete and incomplete data. The number of samples $N$ are chosen to be 100 equidistant values in the log scale between 15 and $50 n$.

- For each pair $(N, p)$ we compute estimation error and average over 100 trials.

The averaged estimation errors are plotted in Figure 1. The black line in the figure corresponds to the estimation error of the sample covariance matrix, and serves as our baseline performance. Theorem 1 indicates that for $N$ large enough, the MCAR estimator with missing data has error $\mathcal{O}\left((p \sqrt{N})^{-1}\right)$, thus to achieve the same error as the sample covariance matrix, it needs more samples by a factor of $p^{-1}$. We verify this experimentally by multiplying the estimation error of the sample covariance matrix by $p^{-1}$. Those curves are shown in dotted lines, and are always above the estimation error of both estimators, almost parallel and tighter when $N / n<1$. Additionally, when $\mathbf{P}$ is unknown, (5) always outperforms (3), even though the latter has access to the actual missing data distribution through $\mathbf{P}$. This might occur because (5) computes exact averages (using $\widehat{\mathbf{P}}$ ), while (3) employs an estimate of the number of observations (a function of $\mathbf{P}$ ).

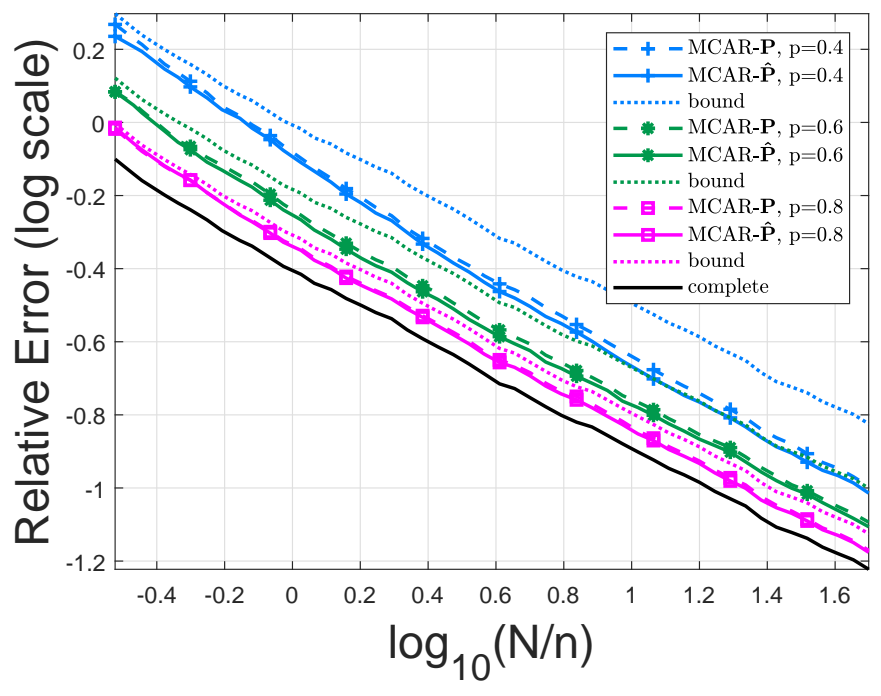

Fig. 1: Comparison of proposed estimators under MCAR missing observations with uniform observation probabilities. The plot shows relative estimation error in operator norm versus number of i.i.d. realizations, both in logarithmic scale. Solid and dashed lines denote estimators (3) (MCAR- $\widehat{\mathbf{P}}$ ) and (5) (MCAR-P) respectively. Dotted lines are an estimate based on the error bounds.

2) Non uniform MCAR observations: In the next experiment we show that when the missing observations have a favorable distribution, not much information is lost due to missing data. We consider a non-singular population covariance matrix $\boldsymbol{\Sigma}$ of dimension $n=50$, and effective rank $\mathrm{r}=2.7472$ (see Figure 2). The missing data distribution is MCAR with independent entries, and observation probabilities given by $\mathbf{p}=\max (\rho \sqrt{\operatorname{diag}(\boldsymbol{\Sigma})}, 1)$. In this setting the variable $x_{i}$ is observed with probability proportional to its standard deviation $\sqrt{\Sigma_{i i}}$. The parameter $\rho$ is set so that the expected number of observed variables are $\{28 \%, 40 \%, 50 \%\}$. The performance of (5) is depicted in Figure 3. It can be seen that when roughly $50 \%$ or more variables are observed in average, the estimator has almost the same performance as the sample covariance matrix that has access to complete observations. This can be attributed to the fact that variables with lower variance contribute less to the estimation error, and thus a higher missing data rate can be tolerated for those entries. 

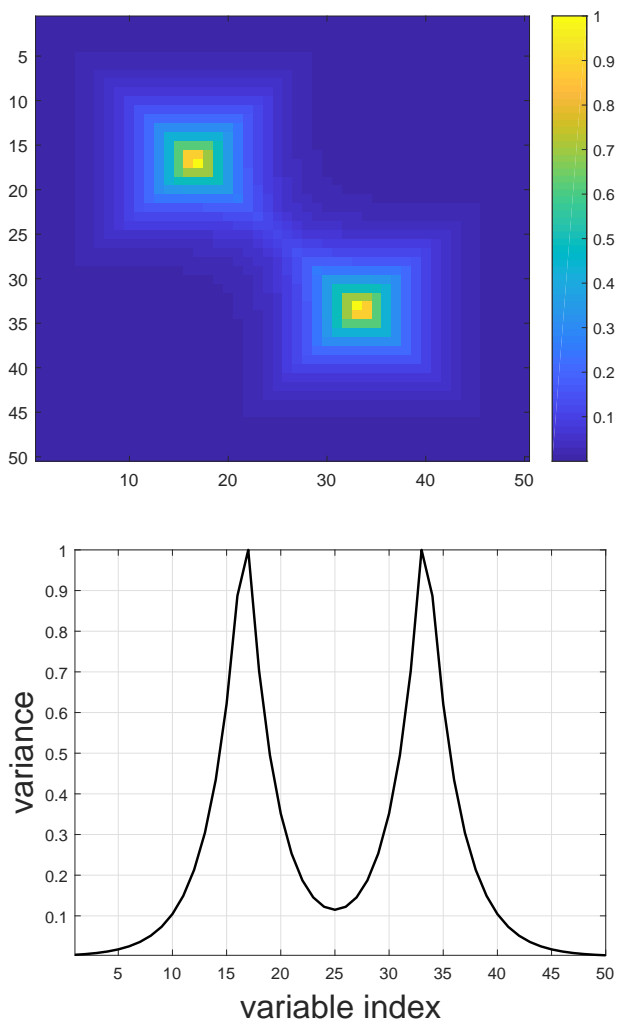

(b) variance

Fig. 2: Approximately sparse covariance matrix with low effective rank.

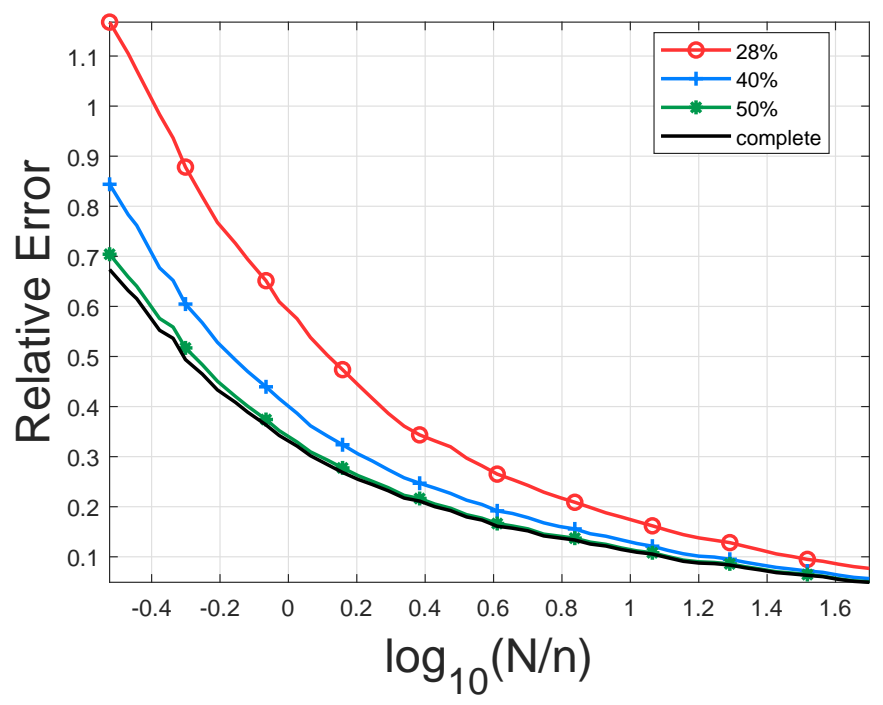

Fig. 3: Evaluation of the estimators under MCAR missing observations with non uniform observation probabilities.

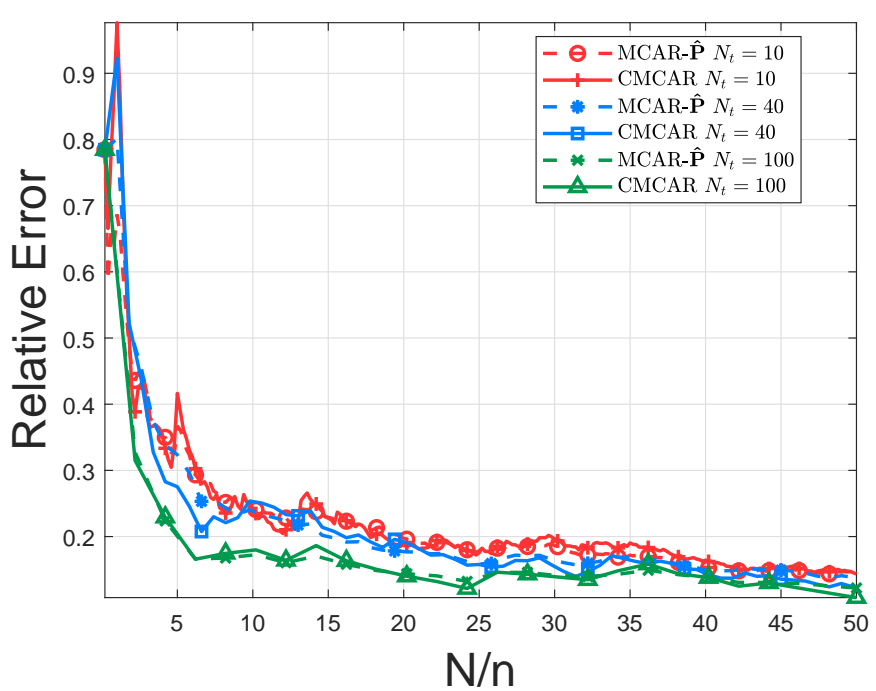

Fig. 4: Comparison of CMCAR and MCAR estimators under time varying missing observations with uniform observation probabilities. The plotted curve shows relative estimation error (in $\log$ scale) in operator norm versus number of i.i.d. realizations. $N_{t}$ is the block size, i.e., the number of realizations until the missing data distribution changes.

\section{B. Estimation under CMCAR observations}

This section compares the estimators from (5) and (7), designed for MCAR and CMCAR observations respectively. We consider a simple CMCAR mechanism, where

$$
\mathbb{P}\left(\delta_{i}^{(t, k)}=1\right)=\left(1-\alpha_{t}\right)+\alpha_{t} \frac{m}{n},
$$

for fixed $m$ and $\alpha_{t}=0.9(1-1 / \sqrt{t})$. When $t=1$, there are complete observations, and the amount of missing data increases with $t$, to reach an uniform observation probability of $0.9 \mathrm{~m} / \mathrm{n}$. We consider the same synthetic dataset created in Section V-A1, and set $m=0.1 n$. We fix $N_{1}=10$ and the remaining block sizes $N_{t}$ for $t \geq 2$ are set to a constant. Figure 4 depicts the average error in operator norm of both estimators. Note that the CMCAR estimator (7) knows the missing data distribution, while the MCAR estimator (5) does not. When the missing data distribution changes faster (smaller $N_{t}$ ), both estimators require more samples to be accurate. Moreover, when the block size increases, and the missing data distribution changes more slowly, the estimation error decays faster, and both estimators behave similarly.

\section{Proofs}

A. Bounding tools

a) Sub-exponential and sub-Gaussian properties [50]:

- If $z$ is a sub-Gaussian random variable, then

$$
\mathbb{E}\left[|z|^{r}\right]^{1 / r} \leq c\|z\|_{\Psi_{2}} \sqrt{r}, \text { for } r \geq 1 .
$$

- If $z$ and $w$ are sub-Gaussian random variables, then $z^{2}$ and $z w$ are sub-exponential with norms satisfying $\left\|z^{2}\right\|_{\Psi_{1}}=\|z\|_{\Psi_{2}}^{2}$, and $\|z w\|_{\Psi_{1}} \leq\|z\|_{\Psi_{2}}\|w\|_{\Psi_{2}}$.

- Let $y_{1}=\delta_{1} x_{1}$ and $y_{2}=\delta_{2} x_{2}$, where $\delta_{1}, \delta_{2}$ are Bernoulli with parameters $p_{1}$ and $p_{2}$, and $x_{1}, x_{2}$ are sub-Gaussian 
random variables. If $\delta_{1}, \delta_{2}$ are independent of $x_{1}$ and $x_{2}$, then

1) $y_{i}$ is sub-Gaussian and $\left\|y_{i}\right\|_{\Psi_{2}} \leq\left\|x_{i}\right\|_{\Psi_{2}}$.

2) $y_{1}^{2}, y_{2}^{2}$, and $y_{1} y_{2}$ are sub-exponential with norms satisfying $\left\|y_{i} y_{j}\right\|_{\Psi_{1}} \leq\left\|x_{i} x_{j}\right\|_{\Psi_{1}}$ for $i, j=1,2$.

b) Useful inequalities:

- Jensen's inequality. Let $z$ be a real random variable, with finite $r$-th moment, then

$$
\mathbb{E}[|z|]^{r} \leq \mathbb{E}\left[|z|^{r}\right], \text { for } r \geq 1 \text {. }
$$

- Application of Jensen's inequality. Let $z_{1}, \cdots, z_{N}$ be non negative random variables with finite $r$-th moments, then

$$
\mathbb{E}\left[\max _{k} z_{k}\right] \leq N^{1 / r} \max _{k} \mathbb{E}\left[z_{k}^{r}\right]^{1 / r}, \text { for } r \geq 1 .
$$

- Minkowski's inequality (triangular inequality). Given $z_{1}, z_{2}$ with finite $r$-th moments, then for any $r \geq 1$

$$
\mathbb{E}\left[\left|z_{1}+z_{2}\right|^{r}\right]^{1 / r} \leq \mathbb{E}\left[\left|z_{1}\right|^{r}\right]^{1 / r}+\mathbb{E}\left[\left|z_{2}\right|^{r}\right]^{1 / r} .
$$

- For any $a, b \in \mathbb{R},(a+b)^{2} \leq 2\left(a^{2}+b^{2}\right)$.

c) Hadamard products:

- We will need the following identify

$$
\left(\mathbf{M} \odot \mathbf{x} \mathbf{x}^{\top}\right)^{2}=\sum_{i=1}^{n} x_{i}^{2} \mathbf{x} \mathbf{x}^{\top} \odot \mathbf{M}_{i} \mathbf{M}_{i}^{\top},
$$

where $\mathbf{M}_{i}$ is the $i$-th column of $\mathbf{M}$.

- If $\mathbf{A}_{1} \prec \mathbf{A}_{2}$, and $\mathbf{B}$ is positive semi-definite, then

$$
\mathbf{A}_{1} \odot \mathbf{B} \prec \mathbf{A}_{2} \odot \mathbf{B}
$$

where $\mathbf{A} \prec \mathbf{B}$ stands for $0 \prec \mathbf{B}-\mathbf{A}$. In particular we have that

$$
\mathbf{A}_{1} \odot \mathbf{B} \prec\left\|\mathbf{A}_{1}\right\| \mathbf{I} \odot \mathbf{B} .
$$

Some of our proofs rely on an expectation bound from [49], originally devised to certify the performance of the masked covariance estimator. By following Theorem 3.2 and the beginning of the proof of Theorem 3.1 in [49] we state their result in the form most useful for us.

Theorem 4 ([49]). Let $\left\{\mathbf{Z}_{k}\right\}$ be a finite sequence of independent, symmetric, self adjoint random matrices of size $n \times n$, and $n \geq 3$, then

$$
\begin{aligned}
& \mathbb{E}\left[\left\|\sum_{k} \mathbf{Z}_{k}-\mathbb{E}\left[\mathbf{Z}_{k}\right]\right\|^{2}\right]^{1 / 2} \leq \\
& \sqrt{\alpha}\left\|\sum_{k} \mathbb{E}\left[\mathbf{Z}_{k}^{2}\right]\right\|^{1 / 2}+\alpha \mathbb{E}\left[\max _{k}\left\|\mathbf{Z}_{k}\right\|^{2}\right]^{1 / 2},
\end{aligned}
$$

where $\alpha=8 e \log (n)$.

\section{B. Proof of Theorem 1}

Applying Theorem 4 to (3) produces

$$
\begin{aligned}
& \mathbb{E}\left[\|\widehat{\boldsymbol{\Sigma}}-\boldsymbol{\Sigma}\|^{2}\right]^{1 / 2} \leq \sqrt{\frac{8 e \log (n)\left\|\mathbb{E}\left[\left(\boldsymbol{\Gamma} \odot \mathbf{y y}^{\top}\right)^{2}\right]\right\|}{N}} \\
& +\frac{8 e \log (n) \mathbb{E}\left[\max _{k}\left\|\boldsymbol{\Gamma} \odot \mathbf{y}^{(k)} \mathbf{y}^{(k)^{\top}}\right\|^{2}\right]^{1 / 2}}{N} .
\end{aligned}
$$

The bounds from [49] for $\left\|\mathbb{E}\left[\left(\boldsymbol{\Gamma} \odot \mathbf{y y}^{\top}\right)^{2}\right]\right\|$ and $\mathbb{E}\left[\max _{k}\left\|\boldsymbol{\Gamma} \odot \mathbf{y}^{(k)} \mathbf{y}^{(k)^{\top}}\right\|^{2}\right]$ are fairly general. They decouple $\mathbf{y}$ and $\mathbf{y}^{(k)}$ from $\boldsymbol{\Gamma}$, thus they can be greatly improved if we consider their relation for this problem.

a) Bounding the matrix variance (first term): Using (30) and applying expectation we obtain

$$
\mathbb{E}\left[\left(\boldsymbol{\Gamma} \odot \mathbf{y y}^{\top}\right)^{2}\right]=\sum_{i=1}^{n} \mathbb{E}\left[y_{i}^{2} \mathbf{y} \mathbf{y}^{\top}\right] \odot \boldsymbol{\Gamma}_{i} \boldsymbol{\Gamma}_{i}^{\top} .
$$

Using independence, each term in the expected value above can be decomposed as follows

$$
\mathbb{E}\left[y_{i}^{2} \mathbf{y} \mathbf{y}^{\top}\right]=\mathbb{E}\left[x_{i}^{2} \delta_{i} \mathbf{x} \mathbf{x}^{\top} \odot \boldsymbol{\delta} \boldsymbol{\delta}^{\top}\right]=\mathbb{E}\left[x_{i}^{2} \mathbf{x} \mathbf{x}^{\top}\right] \odot \mathbb{E}\left[\delta_{i} \boldsymbol{\delta} \boldsymbol{\delta}^{\top}\right] .
$$

Replacing back in (33) and applying (31) to the Hadamard product of positive semi-definite matrices we have

$$
\begin{aligned}
\mathbb{E}\left[\left(\boldsymbol{\Gamma} \odot \mathbf{y y}^{\top}\right)^{2}\right] & =\sum_{i=1}^{n} \mathbb{E}\left[x_{i}^{2} \mathbf{x} \mathbf{x}^{\top}\right] \odot \mathbb{E}\left[\delta_{i} \boldsymbol{\delta} \boldsymbol{\delta}^{\top}\right] \odot \boldsymbol{\Gamma}_{i} \boldsymbol{\Gamma}_{i}^{\top} \\
& \prec \sum_{i=1}^{n}\left\|\mathbb{E}\left[x_{i}^{2} \mathbf{x} \mathbf{x}^{\top}\right]\right\| \mathbf{I} \odot \mathbb{E}\left[\delta_{i} \boldsymbol{\delta} \boldsymbol{\delta}^{\top}\right] \odot \boldsymbol{\Gamma}_{i} \boldsymbol{\Gamma}_{i}^{\top} .
\end{aligned}
$$

Since the right side is a diagonal matrix, its operator norm is its largest entry, and we can bound the matrix variance as

$$
\begin{aligned}
& \left\|\mathbb{E}\left[\left(\boldsymbol{\Gamma} \odot \mathbf{y y}^{\top}\right)^{2}\right]\right\| \leq \\
& \max _{j \in[n]} \sum_{i=1}^{n}\left\|\mathbb{E}\left[x_{i}^{2} \mathbf{x} \mathbf{x}^{\top}\right]\right\|\left(\mathbb{E}\left[\delta_{i} \boldsymbol{\delta} \boldsymbol{\delta}^{\top}\right] \boldsymbol{\Gamma}_{i} \boldsymbol{\Gamma}_{i}^{\top}\right)_{j j} .
\end{aligned}
$$

The following holds

$$
\left(\mathbb{E}\left[\delta_{i} \boldsymbol{\delta} \boldsymbol{\delta}^{\top}\right] \boldsymbol{\Gamma}_{i} \boldsymbol{\Gamma}_{i}^{\top}\right)_{j j}=\frac{1}{p_{i j}} .
$$

The desired bound is obtained after applying Lemma 2 to bound $\left\|\mathbb{E}\left[x_{i}^{2} \mathbf{x} \mathbf{x}^{\top}\right]\right\|$ (at the end of this section), thus producing

$$
\left\|\mathbb{E}\left[\left(\mathbf{\Gamma} \odot \mathbf{y y}^{\top}\right)^{2}\right]\right\| \leq C^{2}\|\mathbf{\Sigma}\| \max _{j \in[n]} \sum_{i=1}^{n} \frac{\Sigma_{i i}}{p_{i j}},
$$

where $C=4 c^{2} / c_{0}$. When $\delta$ has independent coordinates, we have that $p_{i j}=p_{i i} p_{j j}$ when $i \neq j$, therefore we may use the bound

$$
\left\|\mathbb{E}\left[\left(\boldsymbol{\Gamma} \odot \mathbf{y y}^{\top}\right)^{2}\right]\right\| \leq C^{2} \frac{\|\boldsymbol{\Sigma}\|}{p_{\min }}\left(\sum_{k=1}^{n} \frac{\Sigma_{k k}}{p_{k}}\right),
$$

where $p_{\min }=\min _{i} p_{i i}$.

b) Bounding the maximum spectral norm (second term).: For any $r \geq 1$, applying (29) to $\left\|\boldsymbol{\Gamma} \odot \mathbf{y}^{(k)} \mathbf{y}^{(k)^{\top}}\right\|^{2}$ results in

$$
\mathbb{E}\left[\max _{k}\left\|\boldsymbol{\Gamma} \odot \mathbf{y}^{(k)} \mathbf{y}^{(k)^{\top}}\right\|^{2}\right] \leq N^{1 / r} \mathbb{E}\left[\left\|\boldsymbol{\Gamma} \odot \mathbf{y y}^{\top}\right\|^{2 r}\right]^{1 / r} .
$$

The dependency on $k$ is removed because $\mathbf{y}^{(k)}$ are i.i.d. copies of $\mathbf{y}$. Now we only need to bound $\mathbb{E}\left[\left\|\boldsymbol{\Gamma} \odot \mathbf{y y}^{\top}\right\|^{2 r}\right]$. Since the operator norm is bounded by the Frobenius norm, and $y_{i}^{2} \leq x_{i}^{2}$, we have the inequalities

$$
\left\|\boldsymbol{\Gamma} \odot \mathbf{y y}^{\top}\right\|^{2} \leq\left\|\boldsymbol{\Gamma} \odot \mathbf{y y}^{\top}\right\|_{F}^{2}=\sum_{i, j} \gamma_{i j}^{2} y_{i}^{2} y_{j}^{2} \leq \sum_{i, j} \gamma_{i j}^{2} x_{i}^{2} x_{j}^{2} .
$$


Now we apply the triangular and Cauchy-Schwartz inequalities, followed by Assumption 1, thus

$$
\begin{aligned}
\mathbb{E}\left[\left\|\boldsymbol{\Gamma} \odot \mathbf{y y}^{\top}\right\|^{2 r}\right]^{1 / r} & \leq \sum_{i, j} \gamma_{i j}^{2} \mathbb{E}\left[\left(x_{i}^{2} x_{j}^{2}\right)^{r}\right]^{1 / r} \\
& \leq \sum_{i, j} \gamma_{i j}^{2} \mathbb{E}\left[x_{i}^{4 r}\right]^{1 / 2 r} \mathbb{E}\left[x_{j}^{4 r}\right]^{1 / 2 r} \\
& \leq\left(4 r c^{2} / c_{0}\right)^{2} \sum_{i, j} \gamma_{i j}^{2} \Sigma_{i i} \Sigma_{j j}
\end{aligned}
$$

Replacing $\gamma_{i j}=1 / p_{i j}$, leads to

$$
\mathbb{E}\left[\left\|\boldsymbol{\Gamma} \odot \mathbf{y y}^{\top}\right\|^{2 r}\right]^{1 / r} \leq C^{2} r^{2} \sum_{i=1}^{n} \sum_{j=1}^{n} \frac{\Sigma_{i i} \Sigma_{j j}}{p_{i j}^{2}}
$$

The final bound is obtained by minimizing the function $N^{1 / r} r^{2}$ over $r \geq 1$. This is achieved by setting $r=\log (N) / 2$, since the assumption $\log (N) \geq 2$ guarantees that $r \geq 1$ is satisfied. The desired bound is

$$
\mathbb{E}\left[\max _{k}\left\|\boldsymbol{\Gamma} \odot \mathbf{y}^{(k)} \mathbf{y}^{(k)^{\top}}\right\|^{2}\right] \leq\left(e C \frac{\log (N)}{2}\right)^{2} \sum_{i, j=1}^{n} \frac{\Sigma_{i i} \Sigma_{j j}}{p_{i j}^{2}} .
$$

When $\delta$ has independent coordinates, we may use the bound

$$
\mathbb{E}\left[\max _{k}\left\|\boldsymbol{\Gamma} \odot \mathbf{y}^{(k)} \mathbf{y}^{(k)^{\top}}\right\|^{2}\right] \leq\left(e C \frac{\log (N)}{2} \sum_{i=1}^{n} \frac{\Sigma_{i i}}{p_{i i}^{2}}\right)^{2} .
$$

\section{Proof of Theorem 2}

Since $\mathbb{E}[\widehat{\boldsymbol{\Sigma}} \mid \boldsymbol{\Delta}]=\boldsymbol{\Sigma}_{\mathcal{E}}$, we can apply Theorem 4 to (5),

$$
\begin{aligned}
& \mathbb{E}\left[\left\|\widehat{\boldsymbol{\Sigma}}-\boldsymbol{\Sigma}_{\mathcal{E}}\right\|^{2} \mid \boldsymbol{\Delta}\right]^{1 / 2} \leq \\
& \frac{1}{N} \sqrt{8 e \log (n)\left\|\sum_{k=1}^{N} \mathbb{E}\left[\left(\widehat{\boldsymbol{\Gamma}} \odot \mathbf{y}^{(k)} \mathbf{y}^{(k)^{\top}}\right)^{2} \mid \boldsymbol{\Delta}\right]\right\|} \\
& +\frac{8 e \log (n) \mathbb{E}\left[\max _{k}\left\|\widehat{\boldsymbol{\Gamma}} \odot \mathbf{y}^{(k)} \mathbf{y}^{(k)^{\top}}\right\|^{2} \mid \boldsymbol{\Delta}\right]^{1 / 2}}{N} .
\end{aligned}
$$

We follow a strategy close to the one used in the proof of Theorem 1. Here, the random vectors $\mathbf{y}^{(k)}$ are conditionally independent, given the missing data pattern $\boldsymbol{\Delta}$. In addition, these vectors are not identically distributed, therefore computations are more cumbersome.

a) Bounding the matrix variance: To simplify computations we define $\mathbf{M}^{(k)}=\widehat{\boldsymbol{\Gamma}} \odot \boldsymbol{\delta}^{(k)} \boldsymbol{\delta}^{(k)^{\top}}$, therefore

$$
\widehat{\boldsymbol{\Gamma}} \odot \mathbf{y}^{(k)} \mathbf{y}^{(k)^{\top}}=\mathbf{M}^{(k)} \odot \mathbf{x}^{(k)} \mathbf{x}^{(k)^{\top}} .
$$

Since $\mathbf{M}^{(k)}$ depends only on $\boldsymbol{\Delta}$, we can decouple the data and the missing data quantities. The following equalities are based on the MCAR property, and the i.i.d. assumption on $\mathbf{x}^{(k)}$. We obtain the desired conditional expectation after applying (30), resulting in

$$
\begin{aligned}
& \sum_{k=1}^{N} \mathbb{E}\left[\left(\mathbf{M}^{(k)} \odot \mathbf{x}^{(k)} \mathbf{x}^{(k)^{\top}}\right)^{2} \mid \mathbf{\Delta}\right] \\
& =\sum_{k=1}^{N} \sum_{i=1}^{n} \mathbb{E}\left[x_{i}^{(k)^{2}} \mathbf{x}^{(k)} \mathbf{x}^{(k)^{\top}} \mid \mathbf{\Delta}\right] \odot \mathbf{M}_{i}^{(k)} \mathbf{M}_{i}^{(k)^{\top}} \\
& =\sum_{k=1}^{N} \sum_{i=1}^{n} \mathbb{E}\left[x_{i}{ }^{2} \mathbf{x} \mathbf{x}^{\top} \mid \mathbf{\Delta}\right] \odot \mathbf{M}_{i}^{(k)} \mathbf{M}_{i}^{(k)^{\top}} \\
& =\sum_{i=1}^{n} \mathbb{E}\left[x_{i}{ }^{2} \mathbf{x} \mathbf{x}^{\top}\right] \odot \sum_{k=1}^{N} \mathbf{M}_{i}^{(k)} \mathbf{M}_{i}^{(k)}{ }^{\top} .
\end{aligned}
$$

Now we use (31) to bound the Hadamard product of positive definite matrices, thus we have

$$
\begin{aligned}
& \sum_{k=1}^{N} \mathbb{E}\left[\left(\mathbf{M}^{(k)} \odot \mathbf{x}^{(k)} \mathbf{x}^{(k)^{\top}}\right)^{2} \mid \mathbf{\Delta}\right] \\
& \prec \sum_{i=1}^{n}\left\|\mathbb{E}\left[x_{i}{ }^{2} \mathbf{x} \mathbf{x}^{\top}\right]\right\| \mathbf{I} \odot \sum_{k=1}^{N} \mathbf{M}_{i}^{(k)} \mathbf{M}_{i}^{(k)^{\top}} .
\end{aligned}
$$

The resulting matrix on the right side is diagonal, hence its operator norm can be computed in closed form, and the desired operator norm can be bounded as

$$
\begin{aligned}
& \left\|\sum_{k=1}^{N} \mathbb{E}\left[\left(\mathbf{M}^{(k)} \odot \mathbf{x}^{(k)} \mathbf{x}^{(k)^{\top}}\right)^{2} \mid \mathbf{\Delta}\right]\right\| \\
& \leq\left\|\sum_{i=1}^{n}\right\| \mathbb{E}\left[x_{i}{ }^{2} \mathbf{x} \mathbf{x}^{\top}\right]\left\|\mathbf{I} \odot \sum_{k=1}^{N} \mathbf{M}_{i}^{(k)} \mathbf{M}_{i}^{(k)^{\top}}\right\| \\
& =\max _{j} \sum_{i=1}^{n}\left\|\mathbb{E}\left[x_{i}{ }^{2} \mathbf{x} \mathbf{x}^{\top}\right]\right\|\left(\sum_{k=1}^{N} \mathbf{M}_{i}^{(k)} \mathbf{M}_{i}^{(k)^{\top}}\right)_{j j} .
\end{aligned}
$$

To further simplify the right hand side, we note that

$$
\left(\mathbf{M}_{i}^{(k)} \mathbf{M}_{i}^{(k)^{\top}}\right)_{j j}= \begin{cases}\widehat{\gamma}_{i i}^{2} \delta_{i}^{(k)} & \text { if } j=i \\ \widehat{\gamma}_{i j}^{2} \delta_{i}^{(k)} \delta_{j}^{(k)} & \text { otherwise. }\end{cases}
$$

Thus for $i=j$,

$$
\left(\sum_{k=1}^{N} \mathbf{M}_{i}^{(k)} \mathbf{M}_{i}^{(k)^{\top}}\right)_{i i}=\widehat{\gamma}_{i i}^{2} \sum_{k=1}^{N} \delta_{i}^{(k)}=N \widehat{\gamma}_{i i}^{2} \widehat{p}_{i i}=N \frac{1}{\widehat{p}_{i i}} .
$$

If $i \neq j$ and $(i, j) \in \mathcal{E}$, a similar calculation produces

$$
\left(\sum_{k=1}^{N} \mathbf{M}_{i}^{(k)} \mathbf{M}_{i}^{(k)^{\top}}\right)_{i j}=N \frac{1}{\widehat{p}_{i j}} .
$$

Finally, when $i \neq j$ and $(i, j) \notin \mathcal{E}$, we have that

$$
\left(\sum_{k=1}^{N} \mathbf{M}_{i}^{(k)} \mathbf{M}_{i}^{(k)^{\top}}\right)_{i j}=\sum_{k=1}^{N} \widehat{\gamma}_{i i} \widehat{\gamma}_{i j} \delta_{i}^{(k)} \delta_{j}^{(k)}=0
$$

since $\delta_{i}^{(k)} \delta_{j}^{(k)}=0, \forall k$. We conclude by applying Lemma 2 ,

$$
\begin{aligned}
& \left\|\sum_{k=1}^{N} \mathbb{E}\left[\left(\mathbf{M}^{(k)} \odot \mathbf{x}^{(k)} \mathbf{x}^{(k)^{\top}}\right)^{2} \mid \boldsymbol{\Delta}\right]\right\| \leq \\
& C^{2} N\|\boldsymbol{\Sigma}\| \max _{j} \sum_{i:(i, j) \in \mathcal{E}} \frac{\Sigma_{i i}}{\widehat{p}_{i j}} .
\end{aligned}
$$


b) Bounding the maximum spectral norm: We follow the same strategy as in the proof of Theorem 1 and bound the operator norm by the Frobenius norm. We follow this with Minkowski's inequality leading to the sequence of inequalities

$$
\begin{aligned}
& \mathbb{E}\left[\left\|\mathbf{M}^{(k)} \odot \mathbf{x}^{(k)} \mathbf{x}^{(k)^{\top}}\right\|^{2 r} \mid \boldsymbol{\Delta}\right]^{1 / r} \\
& \leq \sum_{i, j} \mathbb{E}\left[\left|x_{i}^{(k)} x_{j}^{(k)}\right|^{2 r} \mid \boldsymbol{\Delta}\right]^{1 / r}\left|M_{i j}^{(k)}\right|^{2} \\
& =\sum_{i, j} \mathbb{E}\left[\left|x_{i}^{(k)} x_{j}^{(k)}\right|^{2 r}\right]^{1 / r}\left|M_{i j}^{(k)}\right|^{2} \\
& \leq \sum_{(i, j) \in \mathcal{E}} \mathbb{E}\left[\left|x_{i}\right|^{4 r}\right]^{1 / 2 r} \mathbb{E}\left[\left|x_{j}\right|^{4 r}\right]^{1 / 2 r} \frac{1}{\widehat{p}_{i j}^{2}} \\
& \leq\left(4 r c^{2}\right)^{2} \sum_{(i, j) \in \mathcal{E}}\left\|x_{i}\right\|_{\Psi_{2}}^{2}\left\|x_{j}\right\|_{\Psi_{2}}^{2} \frac{1}{\widehat{p}_{i j}^{2}} \\
& \leq r^{2}\left(4 c^{2} / c_{0}\right)^{2} \sum_{(i, j) \in \mathcal{E}} \frac{\Sigma_{i i} \Sigma_{j j}}{\widehat{p}_{i j}^{2}} .
\end{aligned}
$$

The desired bound is obtained after setting $r=\log (N) / 2$.

\section{Proof of Theorem 3}

We will use an inequality due to [52], from where we state the required result in the Lemma below.

Lemma 1. Let $\mathbf{W}_{T}=\sum_{t=1}^{T} \tilde{\mathbf{Z}}_{t}$ be a matrix valued martingale satisfying $\mathbb{E}_{t-1}\left[\tilde{\mathbf{Z}}_{t}\right]=\mathbf{0}$, and $\mathbb{E}\left[\left\|\tilde{\mathbf{Z}}_{t}\right\|^{2}\right] \leq \sigma_{t}^{2}<\infty$, then

$$
\mathbb{E}\left[\left\|\mathbf{W}_{T}\right\|^{2}\right] \leq c \log (n+1) \sum_{t=1}^{T} \sigma_{t}^{2} .
$$

Taking $\tilde{\mathbf{Z}}_{t}=\mathbf{Z}_{t}-N_{t} \boldsymbol{\Sigma}$ we just need to bound $\mathbb{E}\left[\left\|\tilde{\mathbf{Z}}_{t}\right\|^{2}\right]$. We can do that by conditioning on previous observations and applying Theorem 1 , thus leading to

$$
\begin{aligned}
& \mathbb{E}_{t-1}\left[\left\|\tilde{\mathbf{Z}}_{t}\right\|^{2}\right] \leq C^{2}\|\boldsymbol{\Sigma}\|^{2} N_{t}^{2} \times \\
& {\left[\sqrt{\frac{8 e \log (n) \mathrm{r}_{\min }\left(\boldsymbol{\Sigma}, \mathbf{P}^{(t)}\right)}{N_{t}}}+\frac{8 e \log (n) \log \left(N_{t}\right) \mathrm{r}_{2}\left(\boldsymbol{\Sigma}, \mathbf{P}^{(t)}\right)}{N_{t}}\right]^{2}}
\end{aligned}
$$$$
\leq 2 C^{2}\|\boldsymbol{\Sigma}\|^{2} \times
$$$$
\left[8 e \log (n) \mathrm{r}_{\min }\left(\boldsymbol{\Sigma}, \mathbf{P}^{(t)}\right) N_{t}+\left(8 e \log (n) \log \left(N_{t}\right)\right)^{2} \mathrm{r}_{2}{ }^{2}\left(\boldsymbol{\Sigma}, \mathbf{P}^{(t)}\right)\right]
$$

Then $\sigma_{t}^{2}$ is equal to the expectation of the above expression. We conclude by adding terms from $t=1$ to $t=T$, dividing by $\left(\sum_{t=1}^{T} N_{t}\right)^{2}$ and computing square root.

\section{E. Proof of Lemma 2}

Lemma 2. If assumption 1 is true, then

$$
\left\|\mathbb{E}\left[x_{i}{ }^{2} \mathbf{x} \mathbf{x}^{\top}\right]\right\| \leq C^{2} \Sigma_{i i}\|\mathbf{\Sigma}\|
$$

The proof follows from the variational definition of operator norm, the Cauchy-Schwartz inequality and Assumption 1, leading to the sequence of inequalities below

$$
\begin{aligned}
\left\|\mathbb{E}\left[x_{i}{ }^{2} \mathbf{x} \mathbf{x}^{\top}\right]\right\| & =\sup _{\|\mathbf{u}\|_{2}=1} \mathbf{u}^{\top} \mathbb{E}\left[x_{i}{ }^{2} \mathbf{x} \mathbf{x}^{\top}\right] \mathbf{u} \\
& =\sup _{\|\mathbf{u}\|_{2}=1} \mathbb{E}\left[x_{i}{ }^{2}\left(\mathbf{x}^{\top} \mathbf{u}\right)^{2}\right] \\
& \leq \sup _{\|\mathbf{u}\|_{2}=1} \mathbb{E}\left[x_{i}{ }^{4}\right]^{1 / 2} \mathbb{E}\left[\left(\mathbf{x}^{\top} \mathbf{u}\right)^{4}\right]^{1 / 2} \\
& \leq 4 c^{2}\left\|x_{i}\right\|_{\Psi_{2}}^{2} 4 c^{2} \sup _{\|\mathbf{u}\|_{2}=1}\left\|\mathbf{x}^{\top} \mathbf{u}\right\|_{\Psi_{2}}^{2} \\
& \leq\left(4 c^{2} / c_{0}\right)^{2} \Sigma_{i i} \sup _{\|\mathbf{u}\|_{2}=1} \mathbf{u}^{\top} \mathbf{\Sigma} \mathbf{u} \\
& =C^{2} \Sigma_{i i}\|\mathbf{\Sigma}\| .
\end{aligned}
$$

\section{CONCLUSION}

This paper studied covariance estimation with various types of missing observations. We first considered observations missing completely at random (MCAR), where variables are observed according to non uniform Bernoulli random variables, independent of the data. We studied unbiased covariance estimators and obtained new error bounds in operator norm that characterize their performance in terms of the scaled effective rank. These results improve upon previous missing data studies, and are consistent with bounds obtained for the complete observations case. We also introduced the conditionally MCAR missing data mechanism, where the missing data pattern evolves over time and may depend on previous observations. We proposed an unbiased estimator and characterized its estimation error under this setting.

Since in general, the missing data distribution is unknown, the estimator (5) is suitable for most applications. Our error bounds indicate that this estimator achieves the same convergence rate than others that have access to the missing data distribution. Our numerical experiments support this claim.

\section{REFERENCES}

[1] Y. Zhang and J. G. Schneider, "Learning multiple tasks with a sparse matrix-normal penalty," in Advances in Neural Information Processing Systems, 2010, pp. 2550-2558.

[2] J. Schäfer and K. Strimmer, "A shrinkage approach to large-scale covariance matrix estimation and implications for functional genomics," Statistical applications in genetics and molecular biology, vol. 4, no. 1, 2005.

[3] E. Bullmore and O. Sporns, "Complex brain networks: graph theoretical analysis of structural and functional systems," Nature Reviews Neuroscience, vol. 10, no. 3, pp. 186-198, 2009.

-[4] J. Bai and S. Shi, "Estimating high dimensional covariance matrices and its applications," Annals of Economics and Finance, vol. 12, no. 2, pp. 199-215, 2011.

[5] P. Stoica, P. Babu, and J. Li, "Spice: A sparse covariance-based estimation method for array processing," IEEE Transactions on Signal Processing, vol. 59, no. 2, pp. 629-638, 2011.

[6] K. Dabov, A. Foi, V. Katkovnik, and K. Egiazarian, "Bm3d image denoising with shape-adaptive principal component analysis," in SPARS'09-Signal Processing with Adaptive Sparse Structured Representations, 2009.

[7] S. Pyatykh, J. Hesser, and L. Zheng, "Image noise level estimation by principal component analysis," IEEE transactions on image processing, vol. 22, no. 2, pp. 687-699, 2013.

[8] Y. Chen, A. Wiesel, Y. C. Eldar, and A. O. Hero, "Shrinkage algorithms for mmse covariance estimation," IEEE Transactions on Signal Processing, vol. 58, no. 10, pp. 5016-5029, 2010. 
[9] H. E. Egilmez, E. Pavez, and A. Ortega, "Graph learning from data under laplacian and structural constraints," IEEE Journal of Selected Topics in Signal Processing, vol. 11, no. 6, pp. 825-841, 2017.

[10] K. Lounici, "High-dimensional covariance matrix estimation with missing observations," Bernoulli, vol. 20, no. 3, pp. 1029-1058, 2014.

[11] F. Bunea and L. Xiao, "On the sample covariance matrix estimator of reduced effective rank population matrices, with applications to fpca," Bernoulli, vol. 21, no. 2, pp. 1200-1230, 2015.

[12] V. Koltchinskii and K. Lounici, "Concentration inequalities and moment bounds for sample covariance operators," Bernoulli, vol. 23, no. 1, pp. 110-133, 2017.

[13] T. T. Cai and A. Zhang, "Minimax rate-optimal estimation of highdimensional covariance matrices with incomplete data," Journal of multivariate analysis, vol. 150, pp. 55-74, 2016.

[14] S. Park and J. Lim, "Non-asymptotic rate for high-dimensional covariance estimation with non-independent missing observations," Statistics \& Probability Letters, 2019.

[15] R. J. Little and D. B. Rubin, Statistical analysis with missing data. John Wiley \& Sons, 2014, vol. 333.

[16] M. Kolar and E. P. Xing, "Consistent covariance selection from data with missing values," in Proceedings of the 29th International Conference on Machine Learning (ICML), 2012, pp. 551-558.

[17] C. Yang, D. Robinson, and R. Vidal, "Sparse subspace clustering with missing entries," in International Conference on Machine Learning, 2015, pp. 2463-2472.

[18] X. J. Hunt, S. Emrani, I. K. Kabul, and J. Silva, "Multi-task learning with incomplete data for healthcare," in KDD Workshop on Machine Learning for Medicine and Healthcare, 2018. [Online]. Available: https://arxiv.org/abs/1807.02442

[19] A. Gonen, D. Rosenbaum, Y. C. Eldar, and S. Shalev-Shwartz, "Subspace learning with partial information," The Journal of Machine Learning Research, vol. 17, no. 1, pp. 1821-1841, 2016.

[20] Y. Chi, "Nearest subspace classification with missing data," in Signals, Systems and Computers, 2013 Asilomar Conference on. IEEE, 2013, pp. 1667-1671.

[21] Y.-H. Chao, "Compression of signal on graphs with the application to image and video coding," Ph.D. dissertation, University of Southern California, 2017.

[22] P.-L. Loh and M. J. Wainwright, "High dimensional regression with noisy and missing data: provable guarantees with non-convexity," The Annals of Statistics, vol. 40, no. 3, pp. 1637-1664, 2012.

[23] K. Jurczak and A. Rohde, "Spectral analysis of high-dimensional sample covariance matrices with missing observations," Bernoulli, vol. 23, no. 4A, pp. 2466-2532, 2017.

[24] K. Lounici, "Sparse principal component analysis with missing observations," in High dimensional probability VI. Springer, 2013, pp. 327-356.

[25] N. Städler and P. Bühlmann, "Missing values: sparse inverse covariance estimation and an extension to sparse regression," Statistics and Computing, vol. 22, no. 1, pp. 219-235, 2012.

[26] S. Veeramachaneni, F. Demichelis, E. Olivetti, and P. Avesani, "Active sampling for knowledge discovery from biomedical data," in European Conference on Principles of Data Mining and Knowledge Discovery. Springer, 2005, pp. 343-354.

[27] C.-P. Lim, J.-H. Leong, and M.-M. Kuan, "A hybrid neural network system for pattern classification tasks with missing features," IEEE transactions on pattern analysis and machine Intelligence, vol. 27, no. 4 , pp. 648-653, 2005.

[28] M. T. Asif, N. Mitrovic, J. Dauwels, and P. Jaillet, "Matrix and tensor based methods for missing data estimation in large traffic networks," IEEE Transactions on Intelligent Transportation Systems, vol. 17, no. 7, pp. 1816-1825, 2016.

[29] A. Deshpande, C. Guestrin, S. R. Madden, J. M. Hellerstein, and W. Hong, "Model-driven data acquisition in sensor networks," in Proceedings of the Thirtieth international conference on Very large data bases-Volume 30. VLDB Endowment, 2004, pp. 588-599.

[30] N. Gershenfeld, S. Samouhos, and B. Nordman, "Intelligent infrastructure for energy efficiency," Science, vol. 327, no. 5969, pp. 1086-1088, 2010.

[31] M. A. Davenport, P. T. Boufounos, M. B. Wakin, and R. G. Baraniuk, "Signal Processing With Compressive Measurements," IEEE Journal of Selected Topics in Signal Processing, vol. 4, no. 2, pp. 445 - 460, 2010.

[32] G. Dasarathy, P. Shah, B. N. Bhaskar, and R. D. Nowak, "Sketching sparse matrices, covariances, and graphs via tensor products," IEEE Transactions on Information Theory, vol. 61, no. 3, pp. 1373-1388, 2015.
[33] Y. Chen, Y. Chi, and A. J. Goldsmith, "Exact and stable covariance estimation from quadratic sampling via convex programming," IEEE Transactions on Information Theory, vol. 61, no. 7, pp. 4034-4059, 2015.

[34] X. Chen, M. R. Lyu, and I. King, "Toward efficient and accurate covariance matrix estimation on compressed data," in International Conference on Machine Learning, 2017, pp. 767-776.

[35] M. Azizyan, A. Krishnamurthy, and A. Singh, "Extreme compressive sampling for covariance estimation," IEEE Transactions on Information Theory, vol. 64, no. 12, pp. 7613-7635, Dec 2018.

[36] F. Pourkamali-Anaraki and S. Becker, "Preconditioned data sparsification for big data with applications to pca and k-means," IEEE Transactions on Information Theory, vol. 63, no. 5, pp. 2954-2974, 2017.

[37] F. Pourkamali-Anaraki, "Estimation of the sample covariance matrix from compressive measurements," IET Signal Processing, vol. 10, no. 9, pp. 1089-1095, 2016.

[38] F. P. Anaraki and S. Hughes, "Memory and computation efficient pca via very sparse random projections," in Proceedings of the 31st International Conference on Machine Learning (ICML-14), 2014, pp. 1341-1349.

[39] M. Naghshvar and T. Javidi, "Active sequential hypothesis testing," The Annals of Statistics, vol. 41, no. 6, pp. 2703-2738, 2013.

[40] G. Dasarathy, A. Singh, M.-F. Balcan, and J. H. Park, "Active learning algorithms for graphical model selection," in Artificial Intelligence and Statistics (AISTATS), 2016, pp. 1356-1364.

[41] J. Scarlett and V. Cevher, "Lower bounds on active learning for graphical model selection," in The 20th International Conference on Artificial Intelligence and Statistics (AISTATS), 2017.

[42] E. Pavez and A. Ortega, "Active covariance estimation by random sub-sampling of variables," in 2018 IEEE International Conference on Acoustics, Speech and Signal Processing (ICASSP), April 2018, pp. 4034-4038.

[43] B. Marlin, "Missing data problems in machine learning," Ph.D. dissertation, University of Toronto, 2008.

[44] N. M. Laird, "Missing data in longitudinal studies," Statistics in medicine, vol. 7, no. 1-2, pp. 305-315, 1988.

[45] Z. Zheng and B. Padmanabhan, "On active learning for data acquisition," in Data Mining, 2002. ICDM 2003. Proceedings. 2002 IEEE International Conference on. IEEE, 2002, pp. 562-569.

[46] M. Saar-Tsechansky, P. Melville, and F. Provost, "Active feature-value acquisition," Management Science, vol. 55, no. 4, pp. 664-684, 2009.

[47] P. Melville, F. Provost, M. Saar-Tsechansky, and R. Mooney, "Economical active feature-value acquisition through expected utility estimation," in Proceedings of the 1st international workshop on Utility-based data mining. ACM, 2005, pp. 10-16.

[48] S. Chakraborty, J. Zhou, V. Balasubramanian, S. Panchanathan, I. Davidson, and J. Ye, "Active matrix completion," in Data Mining (ICDM), 2013 IEEE 13th International Conference on. IEEE, 2013, pp. 81-90.

[49] R. Y. Chen, A. Gittens, and J. A. Tropp, "The masked sample covariance estimator: an analysis using matrix concentration inequalities," Information and Inference: A Journal of the IMA, vol. 1, no. 1, pp. 2-20, 2012.

[50] R. Vershynin, High Dimensional Probability: An Introduction with Applications in Data Science. Cambridge University Press, 2018.

[51] R. Durrett, Probability: theory and examples. Cambridge university press, 2010.

[52] A. Juditsky and A. S. Nemirovski, "Large deviations of vectorvalued martingales in 2-smooth normed spaces," arXiv preprint arXiv:0809.0813, 2008.

\section{APPENDIX}

\section{A. Covariance estimation with unknown population mean}

In this section we derive unbiased estimators when the population mean $\boldsymbol{\mu}$ is unknown, and the missing data is MCAR. We consider two scenarios:

1) Known missing data distribution: the estimators depend on the observed data $\mathbf{y}^{(k)}$ for $k \in[N]$, the population observation probabilities $\mathbf{p}, \mathbf{P}$, and their entrywise inverses $\gamma$ and $\Gamma$ respectively.

2) Unknown missing data distribution: the estimators depend on the observed data $\mathbf{y}^{(k)}$ for $k \in[N]$, the sample 
estimators for the observation probabilities $\hat{\mathbf{p}}, \widehat{\mathbf{P}}$, and their entrywise inverses $\hat{\gamma}$ and $\widehat{\Gamma}$ respectively.

Some quantities related to the missing data mechanism are given next. The sample observation probabilities

$$
\hat{\mathbf{p}}=\frac{1}{N} \sum_{k=1}^{N} \boldsymbol{\delta}^{(k)} .
$$

The sample observation probability matrix is

$$
\widehat{\mathbf{P}}=\frac{1}{N} \sum_{k=1}^{N} \boldsymbol{\delta}^{(k)} \boldsymbol{\delta}^{(k)^{\top}} .
$$

When $\boldsymbol{\delta}^{(k)}$ has dependent Bernoulli entries, we have that

$$
\begin{aligned}
\mathbb{E}[\widehat{\mathbf{P}}] & =\mathbf{P}, \\
\mathbb{E}[\hat{\mathbf{p}}] & =\mathbf{p}=\operatorname{diag}(\mathbf{P}) .
\end{aligned}
$$

Recall that $\boldsymbol{\Gamma}$ and $\widehat{\Gamma}$ are the entry-wise inverses of $\mathbf{P}$ and $\widehat{\mathbf{P}}$ respectively. The entry-wise inverses of $\mathbf{p}$ and $\widehat{\mathbf{p}}$ are defined as $\gamma$ and $\hat{\gamma}$ respectively.

It will also be useful to have estimates for the mean and covariance of the incomplete observations. The sample mean of $\mathbf{y}$ is

$$
\overline{\mathbf{y}}=\frac{1}{N} \sum_{k=1}^{N} \mathbf{y}^{(k)}
$$

This estimator has expectation given by

$$
\mathbb{E}[\overline{\mathbf{y}} \mid \boldsymbol{\Delta}]=\hat{\mathbf{p}} \odot \boldsymbol{\mu} \text {, and } \quad \mathbb{E}[\overline{\mathbf{y}}]=\mathbf{p} \odot \boldsymbol{\mu} .
$$

The covariance of $\mathbf{y}$ can be estimated with

$$
\mathbf{C}_{\mathbf{y}}=\frac{1}{N-1} \sum_{k=1}^{N}\left(\mathbf{y}^{(k)}-\overline{\mathbf{y}}\right)\left(\mathbf{y}^{(k)}-\overline{\mathbf{y}}\right)^{\top} .
$$

When $\mathbf{P}$ is known, $\mathbf{C}_{\mathbf{y}}$ is unbiased for $\operatorname{Cov}(\mathbf{y})$, thus

$$
\mathbb{E}\left[\mathbf{C}_{\mathbf{y}}\right]=\boldsymbol{\Sigma} \odot \mathbf{P}+\left(\mathbf{P}-\mathbf{p p}^{\top}\right) \odot \boldsymbol{\mu} \boldsymbol{\mu}^{\top}=\operatorname{Cov}(\mathbf{y}) .
$$

Note that since the goal is to estimate $\boldsymbol{\Sigma}$, we could attempt to correct $\mathbf{C}_{\mathbf{y}}$ by removing the effect of $\mathbf{P}$ and $\boldsymbol{\mu}$. A simpler strategy is to build estimators using $\overline{\mathbf{y}}$, and

$$
\mathbf{R}_{\mathbf{y}}=\frac{1}{N} \sum_{k=1}^{N} \mathbf{y}^{(k)} \mathbf{y}^{(k)^{\top}} .
$$

Before deriving the covariance estimators, we introduce some useful properties of $\overline{\mathbf{y}}$ and $\mathbf{R}_{\mathbf{y}}$. Proofs are provided in the subsequent appendix.

Lemma 3. The quantity $\mathbf{R}_{\mathbf{y}}$ obeys

$$
\begin{aligned}
\mathbb{E}\left[\mathbf{R}_{\mathbf{y}} \mid \boldsymbol{\Delta}\right] & =\widehat{\mathbf{P}} \odot\left(\boldsymbol{\Sigma}+\boldsymbol{\mu} \boldsymbol{\mu}^{\top}\right) \\
\mathbb{E}\left[\mathbf{R}_{\mathbf{y}}\right] & =\mathbf{P} \odot\left(\boldsymbol{\Sigma}+\boldsymbol{\mu} \boldsymbol{\mu}^{\top}\right) .
\end{aligned}
$$

Lemma 4. When $\mathbf{P}$ is known we have

$\mathbb{E}\left[\overline{\mathbf{y}} \overline{\mathbf{y}}^{\top}\right]=\frac{1}{N}\left(\boldsymbol{\Sigma} \odot \mathbf{P}+\left(\mathbf{P}-\mathbf{p} \mathbf{p}^{\top}\right) \odot \boldsymbol{\mu} \boldsymbol{\mu}^{\top}\right)+\mathbf{p} \mathbf{p}^{\top} \odot \boldsymbol{\mu} \boldsymbol{\mu}^{\top}$.

Lemma 5. When $\mathbf{P}$ is unknown we have

$$
\mathbb{E}\left[\overline{\mathbf{y}} \overline{\mathbf{y}}^{\top} \mid \boldsymbol{\Delta}\right]=\hat{\mathbf{p}} \hat{\mathbf{p}}^{\top} \odot \boldsymbol{\mu} \boldsymbol{\mu}^{\top}+\frac{1}{N} \widehat{\mathbf{P}} \odot \boldsymbol{\Sigma} .
$$

1) Covariance estimation with known $\mathbf{P}$ : According to Lemma $3, \mathbf{R}_{\mathbf{y}} \odot \boldsymbol{\Gamma}$ is an unbiased estimator for $\boldsymbol{\Sigma}+\boldsymbol{\mu} \boldsymbol{\mu}^{\top}$. Our strategy is thus to find an unbiased estimator for $\boldsymbol{\mu} \boldsymbol{\mu}^{\top}$ and subtract it from $\mathbf{R}_{\mathbf{y}} \odot \boldsymbol{\Gamma}$. Since Lemma 3 and 4 imply

$$
\mathbb{E}\left[N^{2} \overline{\mathbf{y}} \overline{\mathbf{y}}^{\top}-N \mathbf{R}_{\mathbf{y}}\right]=N(N-1) \mathbf{p} \mathbf{p}^{\top} \odot \boldsymbol{\mu} \boldsymbol{\mu}^{\top},
$$

an unbiased estimator for $\boldsymbol{\mu} \boldsymbol{\mu}^{\top}$ is given by

$$
\frac{1}{N-1}\left(N \overline{\mathbf{y}} \overline{\mathbf{y}}^{\top}-\mathbf{R}_{\mathbf{y}}\right) \odot \boldsymbol{\gamma} \boldsymbol{\gamma}^{\top} .
$$

Therefore, an unbiased estimator for $\boldsymbol{\Sigma}$ is

$$
\mathbf{R}_{\mathbf{y}} \odot \boldsymbol{\Gamma}-\frac{1}{N-1}\left(N \overline{\mathbf{y}} \overline{\mathbf{y}}^{\top}-\mathbf{R}_{\mathbf{y}}\right) \odot \boldsymbol{\gamma} \boldsymbol{\gamma}^{\top}
$$

2) Covariance estimation with unknown $\mathbf{P}$ : We can derive an estimator following a similar procedure, however here we estimate $\boldsymbol{\mu} \boldsymbol{\mu}^{\top}$ differently. The proposed covariance estimator corresponds to

$$
\mathbf{R}_{\mathbf{y}} \odot \widehat{\boldsymbol{\Gamma}}-\left(N \overline{\mathbf{y}} \overline{\mathbf{y}}^{\top}-\mathbf{R}_{\mathbf{y}}\right) \odot \mathbf{\Psi} .
$$

Where $\Psi$ is the Hadamard inverse of $\Theta=N \hat{\mathbf{p}} \hat{\mathbf{p}}^{\top}-\hat{\mathbf{p}}$. Unbiasedness can be verified by applying Lemma 3 and 5 . A necessary condition for $\Theta$ to be Hadamard invertible is that $\sum_{k=1}^{N} \delta_{i}^{(k)} \geq 2$ for all $i \in[n]$. This result is proven in the next appendix.

3) Comparison with complete observations: Note that when there is no missing data, both covariance estimators from this section become

$$
\frac{1}{N-1} \sum_{k=1}^{N}\left(\mathbf{y}^{(k)}-\overline{\mathbf{y}}\right)\left(\mathbf{y}^{(k)}-\overline{\mathbf{y}}\right)^{\top} .
$$

\section{B. Additional proofs}

1) Proof of Lemma 4: We may use $\boldsymbol{\mu}_{\mathbf{y}}=\mathbf{p} \odot \boldsymbol{\mu}$, then

$$
\begin{aligned}
& N^{2} \overline{\mathbf{y}} \overline{\mathbf{y}}^{\top}=\sum_{k=1}^{N} \sum_{l=1}^{N} \mathbf{y}^{(k)} \mathbf{y}^{(l)^{\top}} \\
& =\sum_{k=1}^{N} \sum_{l=1}^{N}\left(\mathbf{y}^{(k)}-\boldsymbol{\mu}_{\mathbf{y}}\right)\left(\mathbf{y}^{(l)}-\boldsymbol{\mu}_{\mathbf{y}}\right)^{\top}+N^{2} \boldsymbol{\mu}_{\mathbf{y}} \boldsymbol{\mu}_{\mathbf{y}}^{\top} \\
& +\sum_{k=1}^{N} \sum_{l=1}^{N}\left[\left(\mathbf{y}^{(k)}-\boldsymbol{\mu}_{\mathbf{y}}\right) \boldsymbol{\mu}_{\mathbf{y}}^{\top}+\boldsymbol{\mu}_{\mathbf{y}}\left(\mathbf{y}^{(k)}-\boldsymbol{\mu}_{\mathbf{y}}\right)^{\top}\right] \\
& =N \mathbf{S}_{\mathbf{y}}+2 \sum_{k>l}^{N}\left(\mathbf{y}^{(k)}-\boldsymbol{\mu}_{\mathbf{y}}\right)\left(\mathbf{y}^{(l)}-\boldsymbol{\mu}_{\mathbf{y}}\right)^{\top}+N^{2} \boldsymbol{\mu}_{\mathbf{y}} \boldsymbol{\mu}_{\mathbf{y}}^{\top} \\
& +N\left(\overline{\mathbf{y}}-\boldsymbol{\mu}_{\mathbf{y}}\right) \boldsymbol{\mu}_{\mathbf{y}}^{\top}+N \boldsymbol{\mu}_{\mathbf{y}}\left(\overline{\mathbf{y}}-\boldsymbol{\mu}_{\mathbf{y}}\right)^{\top} .
\end{aligned}
$$

We conclude by applying expectation and using the fact that $\mathbb{E}\left[\mathbf{S}_{\mathbf{y}}\right]=\operatorname{Cov}(\mathbf{y})$.

2) Proof of Lemma 5: We have the identity

$$
\begin{aligned}
N^{2} \overline{\mathbf{y}} \overline{\mathbf{y}}^{\top} & =\sum_{k=1}^{N} \sum_{l=1}^{N} \mathbf{y}^{(k)} \mathbf{y}^{(l)}{ }^{\top} \\
& =\sum_{k \neq l} \mathbf{y}^{(k)} \mathbf{y}^{(l)^{\top}}+\sum_{k=1}^{N} \mathbf{y}^{(k)} \mathbf{y}^{(k)^{\top}} .
\end{aligned}
$$


Applying conditional expectation leads to

$$
\begin{aligned}
& \mathbb{E}\left[N^{2} \overline{\mathbf{y}} \overline{\mathbf{y}}^{\top} \mid \boldsymbol{\Delta}\right]=\sum_{k \neq l} \boldsymbol{\delta}^{(k)} \boldsymbol{\delta}^{(l)^{\top}} \odot \boldsymbol{\mu} \boldsymbol{\mu}^{\top} \\
& +\sum_{k=1}^{N} \boldsymbol{\delta}^{(k)} \boldsymbol{\delta}^{(k)^{\top}} \odot\left(\boldsymbol{\Sigma}+\boldsymbol{\mu} \boldsymbol{\mu}^{\top}\right) \\
& =\sum_{k=1}^{N} \sum_{l=1}^{N} \boldsymbol{\delta}^{(k)} \boldsymbol{\delta}^{(l)} \top^{\top} \odot \boldsymbol{\mu} \boldsymbol{\mu}^{\top}-\sum_{k=1}^{N} \boldsymbol{\delta}^{(k)} \boldsymbol{\delta}^{(k)^{\top}} \odot \boldsymbol{\mu} \boldsymbol{\mu}^{\top} \\
& +\sum_{k=1}^{N} \boldsymbol{\delta}^{(k)} \boldsymbol{\delta}^{(k)^{\top}} \odot\left(\boldsymbol{\Sigma}+\boldsymbol{\mu} \boldsymbol{\mu}^{\top}\right) \\
& =N^{2} \hat{\mathbf{p}} \hat{\mathbf{p}}^{\top} \odot \boldsymbol{\mu} \boldsymbol{\mu}^{\top}+N \widehat{\mathbf{P}} \odot \boldsymbol{\Sigma} .
\end{aligned}
$$

3) Hadamard invertibility of $\Theta$ : The $i j$ entry of $\Theta$ obeys

$$
N \theta_{i j}=\sum_{k=1}^{N} \delta_{i}^{(k)} \sum_{k=1}^{N} \delta_{j}^{(k)}-\sum_{k=1}^{N} \delta_{i}^{(k)} \delta_{j}^{(k)} \geq 0 .
$$

When $i=j$, a necessary and sufficient condition for invertibility is that for all $i \in[n], \sum_{k=1}^{N} \delta_{i}^{(k)} \geq 1$. For $i \neq j, \theta_{i j}>0$ if any of the following conditions holds

1) $\sum_{k=1}^{N} \delta_{i}^{(k)}=\sum_{k=1}^{N} \delta_{j}^{(k)}=1$, and $\delta_{i}^{(k)} \delta_{j}^{(k)}=0$ for all $k$,

2) $\sum_{k=1}^{N} \delta_{i}^{(k)} \geq 1$ and $\sum_{k=1}^{N} \delta_{j}^{(k)} \geq 2$,

3) $\sum_{k=1}^{N} \delta_{i}^{(k)} \geq 2$ and $\sum_{k=1}^{N} \delta_{j}^{(k)} \geq 1$.

Case 1) can be verified directly. Case 2) and 3) can be proven using the same argument. Let us consider case 2) first. Since $0 \leq \delta_{j}^{(k)} \leq 1$ we have that

$$
\sum_{k=1}^{N} \delta_{i}^{(k)} \delta_{j}^{(k)} \leq \sum_{k=1}^{N} \delta_{i}^{(k)},
$$

which combined with $\sum_{k=1}^{N} \delta_{j}^{(k)}>1$ implies that $\theta_{i j}>0$. 